\title{
Antibody Dynamics Simulation -Theory and Application
}

\section{Zhaobin Xu ( $\nabla$ zhaobin23@126.com )}

Dezhou University

Dongying Yang

Dezhou University

Hongmei Zhang

Dezhou University

\section{Research Article}

Keywords: sars-cov-2, antibody dynamics, vaccine, protection time, mathematical modeling

Posted Date: October 15th, 2021

DOl: https://doi.org/10.21203/rs.3.rs-967878/v1

License: (1) This work is licensed under a Creative Commons Attribution 4.0 International License. Read Full License 


\title{
Antibody Dynamics Simulation -Theory and Application
}

\author{
Zhaobin $\mathrm{Xu}^{1 *}$, Dongying Yang ${ }^{2}$, Hongmei Zhang ${ }^{1}$ \\ 1 Department of Life Science, Dezhou University, ShanDong, P.R. China, 253023 \\ 2 School of Medicine, Dezhou University, ShanDong, P.R. China, 253023 \\ * Corresponding address: zhaobin23@126.com;
}

\begin{abstract}
Infectious disease such as COVID-19 poses a considerable threat to public health when a pandemic strain emerges. Constructing a reliable mathematical model helps us quantitatively explain the kinetic characteristics of antibody-virus interactions, which could provide a reasonable prediction toward many sensitive concerns faced by the public, such as how to calculate protection time provided by the specific vaccine. A novel and robust model is developed to integrate antibody dynamics with virus dynamics in the host body. Our model is based on a comprehensive understanding of immunology principles rather than a simple data-fitting attempt by arbitrarily mathematical function selection. The physical-based mechanism would bring this model more reliable and broader prediction performance. This model gives quantitative insights between antibody dynamics and virus loading in the host body. Based on this model, we can estimate the antibody dynamic parameters with high fidelity. We could solve lots of critical problems, such as the calculation of vaccine protection time. We can also explain lots of mysterious phenomena such as antibody inferences, self-reinfection, chronic infection, etc. We suggest the best strategy in prolonging the vaccine protection time is not repeated inoculation but a directed induction of fast binding antibodies. Eventually, it will also inform the future construction of the mathematical model and help us fight against those infectious diseases.
\end{abstract}

Keywords: sars-cov-2, antibody dynamics, vaccine, protection time, mathematical modeling

\section{Introduction}

The sars-cov-2 epidemic has caused more than 230 million infections and over 4.75 million deaths worldwide, and it will be difficult to disappear in a short time based on current trends [1-3]. Vaccination has become an excellent approach to fighting COVID-19 infections. As vaccination efforts have become widespread, we better understand the antibodies produced by vaccines or natural infections. It is gradually realized that sars-cov- 2 antibodies, whether acquired from natural infection or triggered by vaccination, decay over time, with a concomitant decrease in protective efficiency. Unlike antibodies produced by vaccinations such as smallpox, these antibodies do not provide durable protection [4]. According to public opinion polls, a significant portion of the population is reluctant to be vaccinated. Of the many reasons, two are the most important. The first is that the hesitation group is concerned about the side effects of vaccination, such as blood clots, etc. $[5,6]$. This problem is relatively easy to handle, and we can solve it by controlling the dose of the vaccine or by using a safer vaccine. Nevertheless, there is a lack of a valid mathematical model to predict the possibility of side effects or optimize the optimal vaccination dose. The second is that many people believe that vaccines do not provide long-lasting protection and, not only that, they fear that the body will show less immunity and become more fragile to mutant strains after vaccination in the long term. The second question is more difficult to answer because, based on current statistical data, we have observed a significant decrease in the immune effect of the vaccine against mutant strains. At present, it still shows an overall positive effect on protection, and the magnitude of the mutation of the virus must increase further with time, so whether the neutralizing antibodies induced by this vaccine will maintain a positive protective power is still an open question. It is essential to answer this question regarding whether vaccination is warranted from a long-term perspective. We do not have access to future clinical data. We need to give predictions on this issue because individual cases of vaccination against some infectious diseases have shown that 
vaccinated individuals may exhibit higher mortality over time and that vaccination with some vaccines may interfere with the efficacy of other vaccines. [7-8] Also, as the issue of the duration of vaccine protection has emerged, more people are concerned about how long the protection can be provided by the antibodies triggered by their immune response $[4,9]$.

Constructing a reliable mathematical model helps us quantitatively explain the kinetic characteristics of antibody-virus interactions, which could provide a reasonable prediction toward those sensitive concerns faced by the public. Researchers have conducted many studies on this problem in terms of mathematical modeling [10-21].

Specifically, depending on whether the immune response is integrated into the models, traditional models can be divided into two categories; the first sets are models that do not take the immune response into account [10-14], that is, traditional viral kinetic models, which the following equation can be classically expressed

$$
\begin{aligned}
& \frac{d T}{d t}=s T-d T-\beta T V ; \\
& \frac{d I}{d t}=\beta T V-\delta \mathrm{I} \\
& \frac{d V}{d t}=p I-c V ;
\end{aligned}
$$

Where $\boldsymbol{T}$ denotes the target cells or the number of susceptible cells, $\boldsymbol{I}$ denotes the number of cells where the infection has occurred, and $\boldsymbol{V}$ denotes the number of viruses. There are two critical disadvantages to this model. The first is that it does not correlate with the body's immune response, and therefore does not provide more accurate information on viral changes. The second problem is that forcing the data fit inevitably leads to underestimating the value $\boldsymbol{T}_{\boldsymbol{0}}$. This model assumes that the driving force behind the virus increase, decay, and elimination process is due to the depletion of susceptible cells. This assumption is incorrect because biologically speaking, the number of susceptible cells is a significant value, including almost all cells in the host body, and even at the moment when the virus is finally eliminated, the number of susceptible cells still does not drop to zero, but remains on a considerable value basis.

The second sets are models that consider the immune response, where the classical expression of the model that considers antibody binding is displayed as follows [15-21].

$$
\begin{aligned}
\frac{d T}{d t} & =\chi D-\beta T V \\
\frac{d I_{1}}{d t} & =\beta T V-k I_{1} \\
\frac{d F}{d t} & =\omega V-\alpha F \\
\frac{d D}{d t} & =\delta I_{2}-\chi D \\
\frac{d I_{2}}{d t} & =k I_{1}-\delta I_{2} \\
\frac{d A}{d t} & =f V-h A \\
\frac{d V}{d t}=\frac{p}{1+\varepsilon_{1} F} & I_{2}-c V-\gamma T V-\kappa V A
\end{aligned}
$$

Where $\boldsymbol{T}$ denotes the overall number of susceptible cells, $\boldsymbol{A}$ denotes the number of antibodies, $\boldsymbol{V}$ denotes the amount of virus, $\boldsymbol{D}$ denotes the number of dead cells, $\boldsymbol{F}$ denotes the amount of interferon in nonspecific immunity, $I_{1}$ denotes the number of cells infected in class 1 , and $I_{2}$ denotes the number of cells infected in class 2 . This model effectively combines antibody dynamics with viral changes. Nevertheless, this model has several noticeable flaws. The first is the lack of physical solid mechanism support, where the idea that antibody change is linearly and positively correlated with virus 
concentration in $\frac{d A}{d t}=f V-h A$ in Eq. (9) lacks biological mechanism support. Also, $\frac{d V}{d t}=$ $\frac{p}{1+\varepsilon_{1} F} I_{2}-c V-\gamma T V-\kappa V A$ in Eq. (10) again lacks a robust biological mechanism to support it. Specifically, the proliferative capacity of the virus is not equal to $\frac{p}{1+\varepsilon_{1} F} I_{2}$, while the $-\gamma T V$ in the third term on the right-hand side of equation (10) also lacks sufficient experimental data to support it. The second drawback of this model is that it cannot consider the kinetic characteristics of antibodies. Different antigen-binding antibodies will exhibit different kinetic characteristics, and this difference in binding affinity or binding activity is an essential factor influencing the kinetic changes of both. Different antibodies will reflect different binding kinetic characteristics while causing different characteristics of viral kinetic changes. However, those conventional models do not take this into account. The third point is also fatal. This model, or this class of models, uses too many compartments. Although multiple compartments can increase the refinement of the model, a significant concern that arises is that it is impossible to solve the parameters with certainty. The increment in parameters sets will display a better fitting performance but at the cost of scarification of prediction reliability. The estimation results are more likely to be locally optimal solutions rather than globally optimal solutions. Such different locally optimal solutions can all exhibit excellent fitting performance, but their prediction curves are very different, making the application of multi-parameter estimation highly unreliable.

To summarize, although models of antibody kinetics have significantly been advanced and developed in recent years, they are still at a highly primitive stage. Therefore, we present here a novel theory of antibody kinetics and apply this model to carry out the prediction of many critical problems and the interpretation of complex phenomena. The model we have developed differs from previous research efforts in several ways.

First: we do not seek the mathematical formula that best fits the experimental data by simply picking a mathematical formula. Instead, we attempted to build a mathematical model that is supported by physical principles or biochemistry principles. We have emphasized the pernicious effect of antibodies on pathogenic microorganisms while noting the activating effect of pathogenic antigens on antibodies based on immunological principles. We present this activating effect in a robust mathematical form.

Second: We have developed a dynamic model that hypothesizes that environmental factors maintain memory cell levels. We consider the natural decay of antibodies while arguing that their decay trend is not continuously maintained all the time. Antigen-like substances in the environment can maintain specific B cells or T cells at certain concentration levels.

Third: We have developed a dynamic model to represent the antigen-antibody binding process. This binding dynamic is described in both the binding process and dissociation, rather than simply using equilibrium constants to calculate the number of antibodies or viruses bound at a given point in time.

Based on the basic principles of immunology, we have established the theoretical hypothesis of antibody kinetics. According to the mechanism description of immunology, although human immunology contains many components and its compartments are incredibly complex, in summary, the core components include two aspects. Those two are the process of antigen recognition with B-cell antibodies generated by germline cell differentiation and the process of B-cell presentation of antigen to T-cells that will promote specific B-cell proliferation [22]. We have represented these two processes in a mathematical model, and the specific principles and processes are given in more detail in the results and methods sections.

Then we realize that if there are certain levels of B cells in the body that can bind to the antigen but with weak binding affinity, it might diminish the opportunity for Germline B cells to come into contact with the antigen. This might decrease the opportunity of the solid binding B cells, which is produced by the differentiation of Germline B cells to proliferate further. As for vaccination, we would like to induce the production of large doses of strongly binding neutralizing antibodies. However, due to differences in B-cell constructions between individuals, individuals who already have weakly binding antibodies in their body may inhibit the production of large amounts of specific antibodies, which is what we define as antibody interference. We next demonstrate utilizing mathematical modeling that antibody interference is likely to be present within our immune system and that this interference may be one of the reasons for the differences in efficiency between individuals after vaccination. Further wet 
experiments can test our hypothesis. For instance, for the experimental group vaccinated with the original antigen, secondary vaccination with the variant antigen produced significantly lower concentrations of specific neutralizing antibodies against the variant antigen than the control group who are not vaccinated with the original antigen and directly vaccinated with the variant antigen.

Given the presence of antibody interference, it is an open question whether vaccinated individuals will exhibit strong or weak immunity to future highly mutated viruses. Because the presence of low-binding neutralizing antibodies in their bodies can eliminate the virus to some extent, but at the same time create a barrier to the proliferation of high-binding neutralizing antibodies, it is still unknown the effect on the overall effect viral load will be. Therefore, we need to use mathematical simulations to quantify how such low-binding antibodies affect the overall change in virus in vivo.

Our antibody kinetic model also answers the following critical and exciting questions.

First: How are memory cells maintained?

Second: How does our immune system screen for antibodies which have a strong binding affinity?

Third: Why do people who get influenza or other vaccines have a lower mortality rate from COVID19 ?

Fourth: How can we effectively calculate the duration of protection of a specific antibody?

Fifth: Why are some of the recovered COVID-19 patients retested as positive cases without infections from other people?

Sixth: How are chronic infections triggered?

Seventh: Why do vaccinations show considerable differences in protection efficiency?

Eighth: How can we improve the protective efficiency and duration of vaccines?

\section{Methods}

\section{Mathematical representation of the antibody production process}

We established the following equation to represent the proliferation process of antibodies

$$
\begin{aligned}
& \frac{d y}{d t}=\left(1+\frac{x}{y}\right)^{n} * y * \alpha-y \\
& \frac{d x}{d t}=k 1 * z *(y-x)-k 2 * x-x ; \\
& \frac{d z}{d t}=\beta *(z-x)-z ;
\end{aligned}
$$

Where $\boldsymbol{x}$ denotes the amount of antibody bound to the antigen, $\boldsymbol{y}$ denotes the total number of antibodies, and $\boldsymbol{z}$ denotes the number of viruses. $\boldsymbol{\alpha}$ denotes the decay coefficient of the antibody, which is related to the half-life of the antibody, and $\boldsymbol{\alpha}$ is a coefficient less than 1. $\boldsymbol{\beta}$ denotes the proliferation coefficient of the virus, which is related to the replication ability of the virus, and the greater the replication ability of the virus, the greater the value of $\boldsymbol{\beta}$, and the value of $\boldsymbol{\beta}$ is a coefficient greater than $1 . \boldsymbol{n}$ is also a constant, which denotes the antigen-like substance stimulatory ability to immune $\mathrm{T}$ cells, the stronger the stimulatory ability, the larger the value of $\boldsymbol{n}$, the greater the proliferation ability of T cells to B cells, and the faster the growth rate of antibodies. $k 1$ denotes the binding constant of the binding reaction between antibody and viral antigen, and $k 2$ denotes the reaction coefficient of the dissociation reaction of the antibody-virus complex. What we need to explain here is equation (12). This equation is different from the following term: $\frac{d x}{d t}=k 1 * z *(y-x)-k 2 * x$ by adding a $-x$ term further. Because according to our theory, the former binding proportion of antibodies denoted as $\mathrm{x}$ will be consumed or depleted by the killing cells. Therefore, the time derivative of $\mathrm{x}$ is different from traditional biochemical reactions.

\section{Mathematical modeling including environmental antigens}




$$
\begin{aligned}
& \frac{d y}{d t}=\left(1+\frac{x}{y}\right)^{n} * y * \alpha+\left(1+\frac{p}{y}\right)^{c} * y * \alpha-y ; \\
& \frac{d x}{d t}=k 1 * z *(y-x-p)-k 2 * x-x ; \\
& \frac{d p}{d t}=k 3 * q *(y-x-p)-k 4 * p-p ; \\
& \frac{d z}{d t}=\beta *(z-x)-z ; \\
& \frac{d q}{d t}=0
\end{aligned}
$$

Where $\boldsymbol{x}$ denotes the number of antibodies bound to the viral antigen; $\boldsymbol{y}$ denotes the total number of antibodies; $\boldsymbol{z}$ denotes the number of viruses; $\boldsymbol{q}$ denotes the amount of antigen-like substances in the environment; $\boldsymbol{p}$ denotes the number of antibodies bound to antigen-like substances in the environment; $\alpha$ denotes the decay coefficient of the antibody, which is related to the half-life of the antibody, $\alpha$ is a coefficient less than $1 ; \boldsymbol{\beta}$ denotes the proliferation coefficient of the virus, which is related to the replication ability of the virus, the greater the replication ability of the virus, the greater the value of $\boldsymbol{\beta}$, $\beta$ is a coefficient greater than 1 . The greater the ability of the virus to replicate, the greater the value of $\boldsymbol{\beta}$, which is a factor greater than $1 . \boldsymbol{n}$ is also a constant, which indicates the stimulating ability of viral antigen-like substances to immune $T$ cells, the stronger the stimulating ability, the greater the value of $\mathrm{n}$, the stronger the proliferation ability of T cells to B cells, and the faster the growth rate of antibodies. $c$ is also a constant, which indicates the stimulating ability of environmental antigen-like substances to immune $\mathrm{T}$ cells. The stronger the stimulating ability, the greater the value of $\boldsymbol{c}$, the greater the proliferative capacity of $\mathrm{T}$ cells to $\mathrm{B}$ cells, and the faster the rate of antibody growth. $k 1$ indicates the binding constant of free antibody and viral antigen, and $k 2$ indicates the reaction coefficient of the dissociation reaction of the antibody-virus complex. $k 3$ indicates the binding constant of free antibody and antigen-like substances in the environment, and $k 4$ indicates the reaction coefficient of the dissociation reaction of the antibody-antigen-like substance complex. In the modeling part, we set $\boldsymbol{c}=$ $\boldsymbol{n}=\mathbf{1}$ for simplicity while admitting that different antigens' stimulating potentials are different. We also set the overall amount of antigen-like substances in the environment as a constant value different from the pathogen dynamics.

\section{A simplified model simulating the proliferation of antibodies with different binding kinetic characteristics by the immune system}

$$
\begin{aligned}
& \frac{d y_{i}}{d t}=\left(1+\frac{x_{i}}{y_{i}}-w_{i}\right)^{c} * y_{i} * \alpha+w_{i}-y_{i} \\
& \frac{d x_{i}}{d t}=\operatorname{Kon}_{i} * z *\left(y_{i}-x_{i}\right)-\operatorname{Koff}_{i} * x_{i}-x_{i} \\
& \frac{d z}{d t}=\beta *\left(z-\sum_{i=1}^{n} x_{i}\right)-z
\end{aligned}
$$

Where xi denotes the amount of the $\boldsymbol{i}$-th antibody that binds to the viral antigen, $y_{i}$ denotes the total number of the $\boldsymbol{i}$ - $\boldsymbol{t} \boldsymbol{h}$ antibody, $\boldsymbol{z}$ denotes the number of viruses, $\boldsymbol{\alpha}$ denotes the decay coefficient of the antibody, and $\boldsymbol{\beta}$ denotes the proliferation coefficient of the virus. $\boldsymbol{c}$ is a constant which indicates the ability of the viral antigen-like substance to stimulate immune T cells; the more potent the stimulation ability, the larger the $c$ value, the more substantial the proliferation of T cells to B cells, and the faster the rate of antibody growth. $\quad w_{i}$ is also a constant that indicates the threshold for maintaining the $\boldsymbol{i}$-th antibody by the antigen-like substance in the environment. $K o n_{i}$ denotes the binding constant of the free antibody $\boldsymbol{i}$ and the viral antigen. $K o f f_{i}$ denotes the reaction coefficient for the dissociation reaction of the antibody $\boldsymbol{i}$ viral complex. 
We use MATLAB to build the system of ordinary differential equations and the simulation solution using the ode15 function.

\section{Parameter estimation using a global searching approach}

In order to estimate the parameters in results 5.3, we developed a simple global searching approach. A least square approach is applied. An objective function is set up as the deviation between experimental data and prediction data. Then a step size is chosen to allow the parameter increase from the lower limit to its upper limit.

The pseudocodes are presented as follows

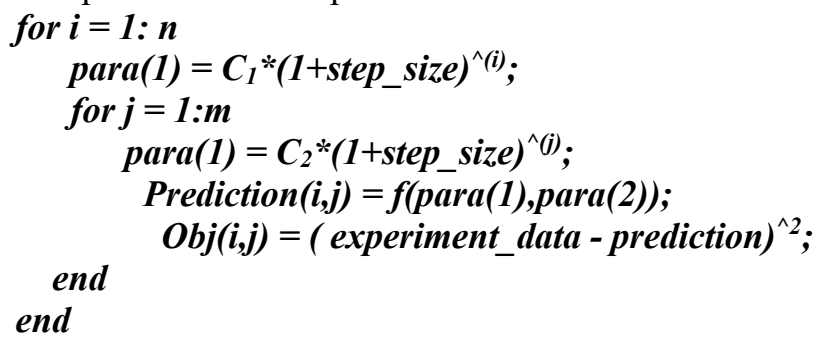

we can obtain the parameter value by seeking $\boldsymbol{i}$ and $\boldsymbol{j}$ with the minimal value of $\boldsymbol{O b j}$.

\section{Results}

\section{Physical mechanism behind this model}

Our antibody model is based on the following four theories.

The first is the reciprocal relationship between the antibody and the pathogen-like substance. The $T$ cells will achieve proliferation of the B cells through the antigen-presenting process, or more directly, the $T$ cells will proliferate the antibody that can bind the antigen. The rate of this stimulation is expressed by equation (11) in the model.

The second is the kinetic relationship between the antibody and the antigen-like substance, and this antigen-antibody binding is represented by the kinetic equation (12).

The third is the pernicious effect of antibodies on pathogenic-like substances, expressed in equation (13).

Fourth, and importantly, we believe that the maintenance of memory cells is dependent on antigen-like substances in the environment. That is, once an antibody is produced, it has a half-life, and after the loss of antigen-like stimulation, there is a gradual decline in the overall concentration of the antibody, but this decay is not always continuous; the rate of decay decreases gradually over time, and the final concentration is maintained at a relatively weak level. Sometimes we refer to the immune cells during this period as memory cells. We believe that the antigen-like substances in the environment stimulate the so-called memory cells that maintain a specific concentration. Because the binding of the antigenlike substances is significantly weaker than that of the original antigen, they can eventually maintain a relatively weak antibody level rather than eventually going away. Thus, we propose there is a presence of a corresponding environmental threshold for a particular antibody in the simulation. This hypothesis could also explain why some antibodies can persist in the body without their concentration decreasing all the time. This mechanism might also contribute to the maintenance of what we usually call memory cells, which are not a specific type of immune cell, but simply a state of equilibrium between their decay and the value added by environmental stimuli at a relatively low concentration, and thus the persistence of such antibodies in the human body. The presence of the maintenance effect of this type of antigen allows us to produce lifelong immunity to some pathogens.

\subsection{Maintenance of memory cells: the environmental antigen maintenance theory}

How memory cells are maintained, and environmental factors stabilize memory cells. Here below, we use an example to illustrate how environmental antigen-like substances help maintain immune cells. 
As described in the methods part, the total number of antibodies is represented as $y$, and the number of antibodies that bind is $\mathrm{x}$. An antibody decay rate is a ubiquitous number which we set $\alpha$ to be 0.98 . The virus proliferation constant $\beta$ is set to be 1.1. when the equilibrium state is reached, the following two equations will be satisfied.

$$
\begin{aligned}
& k 3 *(y-x) *(q-x)=k 4 *(x+1) \\
& \left(1+\frac{x}{y}\right) * \alpha=1
\end{aligned}
$$

Equation (22) stands for the equilibrium state when the binding antibody remain stable.

Equation (23) represents the number of antibodies reaches equilibrium

In turn, the theoretical level of final antibody maintenance can be calculated as

$$
y=\left(q-(k 4+1) \frac{1-\alpha}{k 3(2 \alpha-1)}\right) \frac{\alpha}{1-\alpha} ;
$$

Although the theoretical antibody maintenance levels are difficult to achieve during the actual kinetics, the presence of antigen-like substances in the environment dramatically attenuates the rate of antibody decay. The results of the simulations are shown in figure 1.

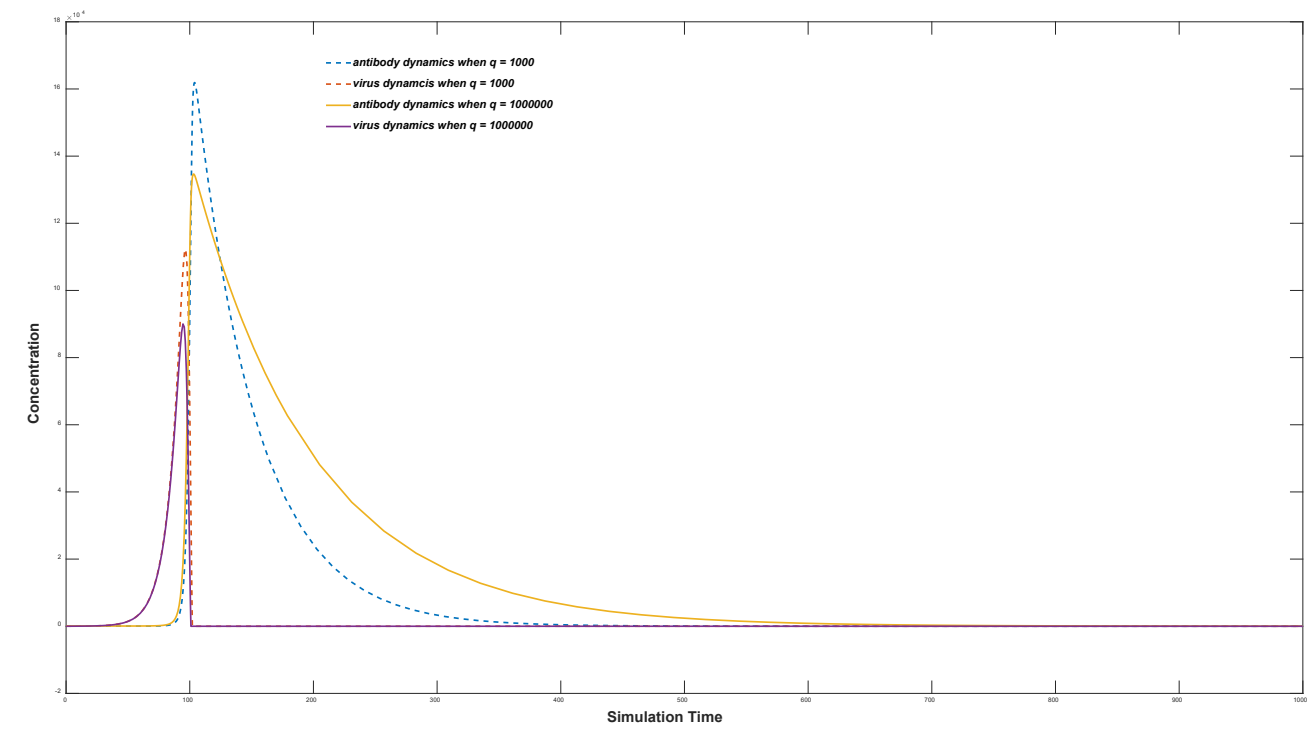

Figure 1A: antibody and virus dynamics modeling using different $q(0)$ value. As shown in figure $1 \mathrm{~A}$, the antibody decay rate is significantly slower (shown in yellow curve) when there is large amount of environmental antigen-like substances. The parameter sets we used are $\mathrm{x}(0)=0, \mathrm{y}(0)=100$, $\mathrm{z}(0)=10, \mathrm{q}(0)=1000$ or $\mathrm{q}(0)=1000000, \mathrm{k} 1=1 \mathrm{e}-5, \mathrm{k} 2=1 \mathrm{e}-14, \mathrm{k} 3=1 \mathrm{e}-8, \mathrm{k} 4=1 \mathrm{e}-14$. 


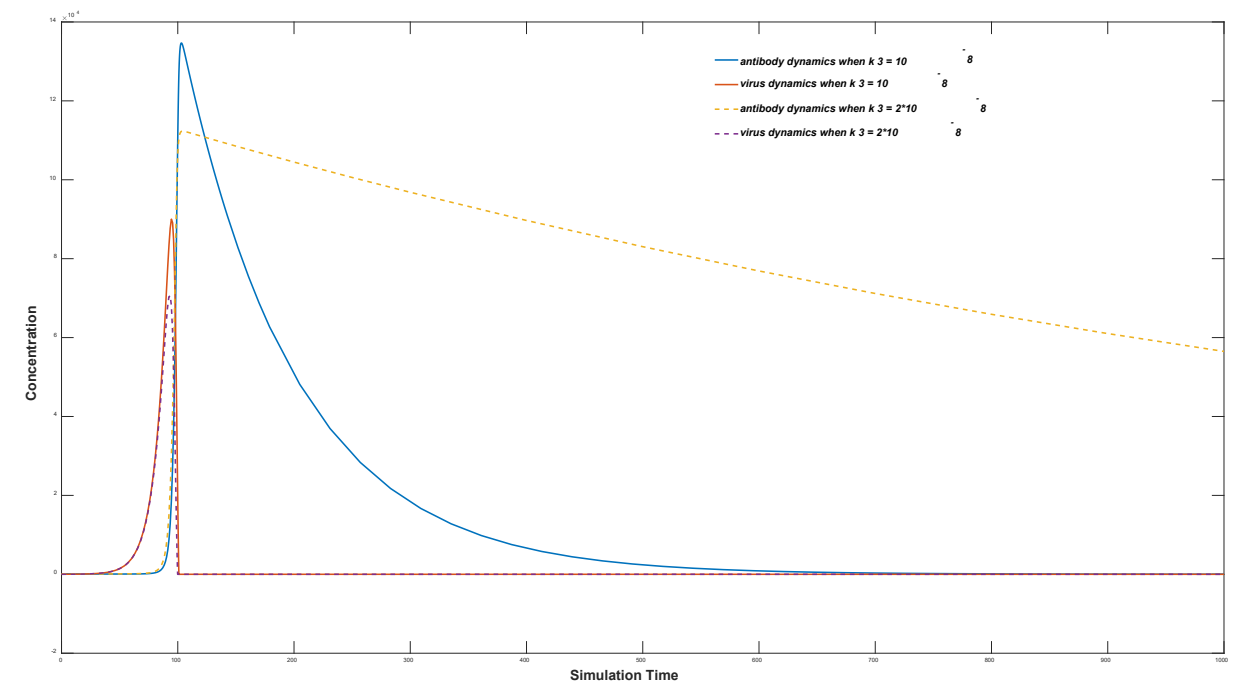

Figure 1B: antibody and virus dynamics modeling using different $q(0)$ value. As shown in figure $1 \mathrm{~B}$, the antibody decay rate is significantly slower (shown in yellow curve) when K3 sets a large value which represents there is a better binding kinetics between environmental antigen-like stuff and its corresponding antibody. The parameter sets we used are $\mathrm{x}(0)=0, \mathrm{y}(0)=100, \mathrm{z}(0)=10, \mathrm{q}(0)=$ $1000000, \mathrm{k} 1=1 \mathrm{e}-5, \mathrm{k} 2=1 \mathrm{e}-14, \mathrm{k} 3=1 \mathrm{e}-8$ or $2 \mathrm{e}-8, \mathrm{k} 4=1 \mathrm{e}-14$.

Therefore, we can conclude that the rate of decay of the antibody depends on the properties of the antibody, which will decay more slowly if it has good binding kinetics to the antigen-like substance in the environment. The decay rate of antibodies is also closely related to the concentration of the antigenlike substance in the environment. The higher the concentration of the substance, the slower the rate of decay.

At the same time, we need to prove that antigen-like substances in the environment cannot stimulate antibody proliferation when a pathogenic antigen does not stimulate the antibody.

The theoretical derivation is as follows, at which point $\mathrm{y}=\mathrm{n}$ ( $\mathrm{n}$ is a small value)

Under the equilibrium state described in equation (22), it is theoretically possible that under some parameters set, $(1+\mathrm{x} / \mathrm{y})^{*} \alpha>1$; when this occurs, the antibody starts to proliferate.

However, kinetic simulations mimicking real scenarios show that antigen-like substances in the environment hardly stimulate antibody proliferation. This situation can be altered when the antigen-like substances are sufficient, or their binding affinities toward some kinds of antibodies are powerful. All those cases are shown in Figure 2. 


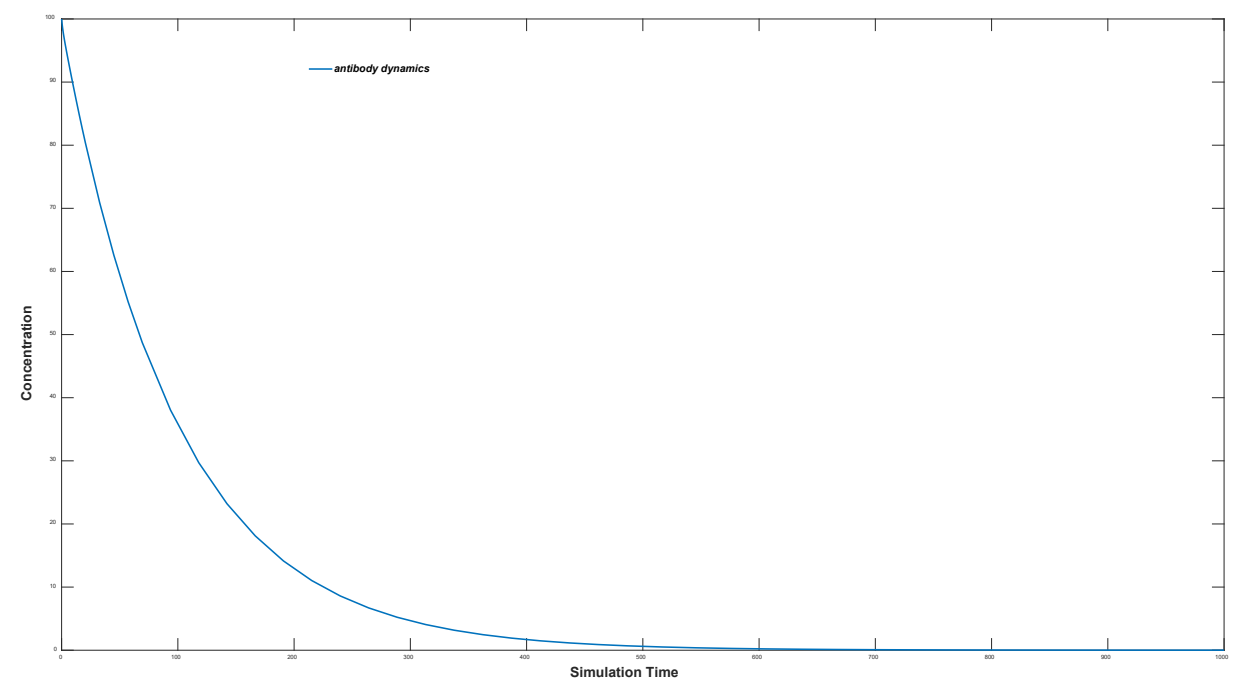

Figure 2A: One scenario that environmental antigen-like substances do not trigger antibody growth.

As shown in figure 2A, the antibody does not engage proliferation due to the presence of environment antigen-like molecules. The parameter sets we used are $\mathrm{x}(0)=0, \mathrm{y}(0)=100, \mathrm{z}(0)=0, \mathrm{q}(0)=1000000$, $\mathrm{k} 1=1 \mathrm{e}-5, \mathrm{k} 2=1 \mathrm{e}-14, \mathrm{k} 3=1 \mathrm{e}-8, \mathrm{k} 4=1 \mathrm{e}-14$.

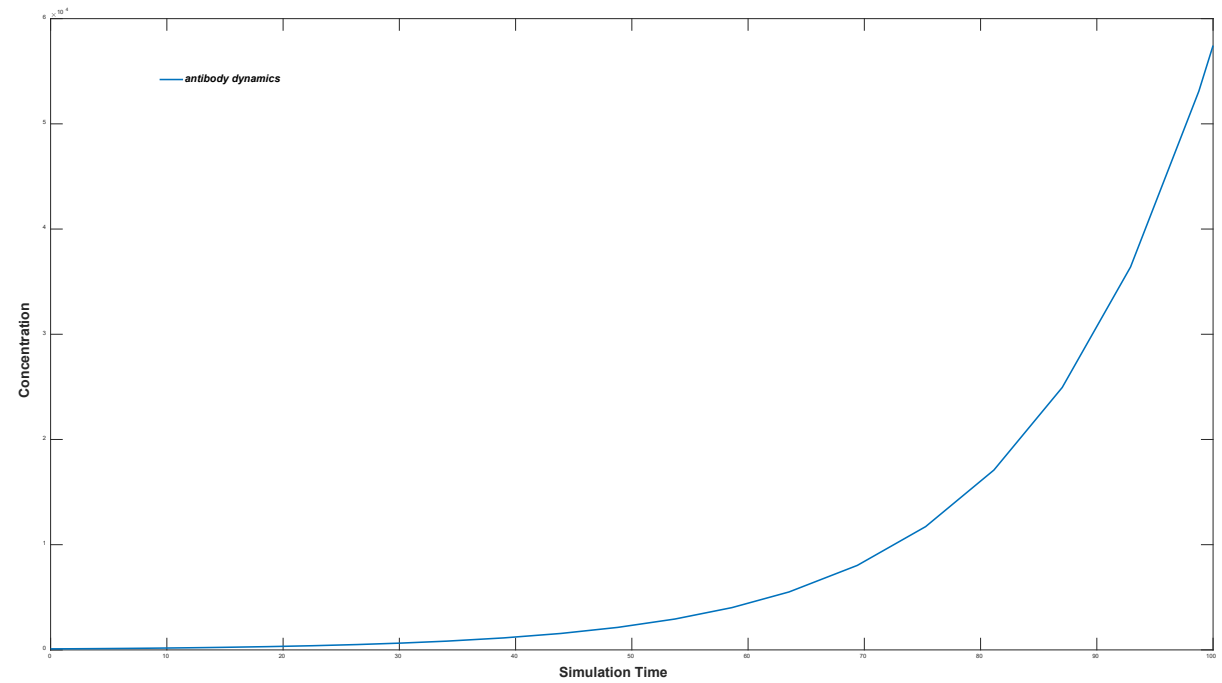

Figure 2B: One scenario that environmental antigen-like substances do trigger antibody proliferation.

As shown in figure $2 \mathrm{~B}$, the antibody does engage proliferation due to the presence of environment antigen-like molecules. The parameter sets we used are $\mathrm{x}(0)=0, \mathrm{y}(0)=100, \mathrm{z}(0)=0, \mathrm{q}(0)=1000000$, $\mathrm{k} 1=1 \mathrm{e}-5, \mathrm{k} 2=1 \mathrm{e}-14, \mathrm{k} 3=1 \mathrm{e}-7, \mathrm{k} 4=1 \mathrm{e}-14$.

For most cases, the presence of antigen-like substances in the environment will not directly stimulate antibody proliferation but will significantly attenuate the rate of decay after antibody proliferation, as shown in figure 2A. However, this is not absolute, as antigen-like substances in the environment can also stimulate antibody levels, as displayed in figure $2 \mathrm{~B}$. This case is why an allergy reaction occurs. The difference between antigen-like substances in the environment and antigen-like substances in 
pathogenic microorganisms is that environmental antigen-like molecules cannot proliferate themselves. However, their levels are maintained at a relatively constant level due to constant replenishment from the environment.

2 Immune response characteristics of immune response after infected with different virus strains Overall, in our experience, highly virulent strains have high replication activity. This type of infection will lead to a high peak infection load and can exhibit high infectivity during the infection cycle due to a high average viral load in the host body. However, the infection period usually is short. Conversely, weak virulent strains have lower replication activity and lower peak viral loads during infection. Since they have lower average viral loads in the body during the infection cycle, they will exhibit weaker infectivity per unit of time. However, this weak virus does not typically induce a quick immune response, resulting in a more extended infection period.

All those situations can be recaptured using our model, which are displayed in Figure 3.

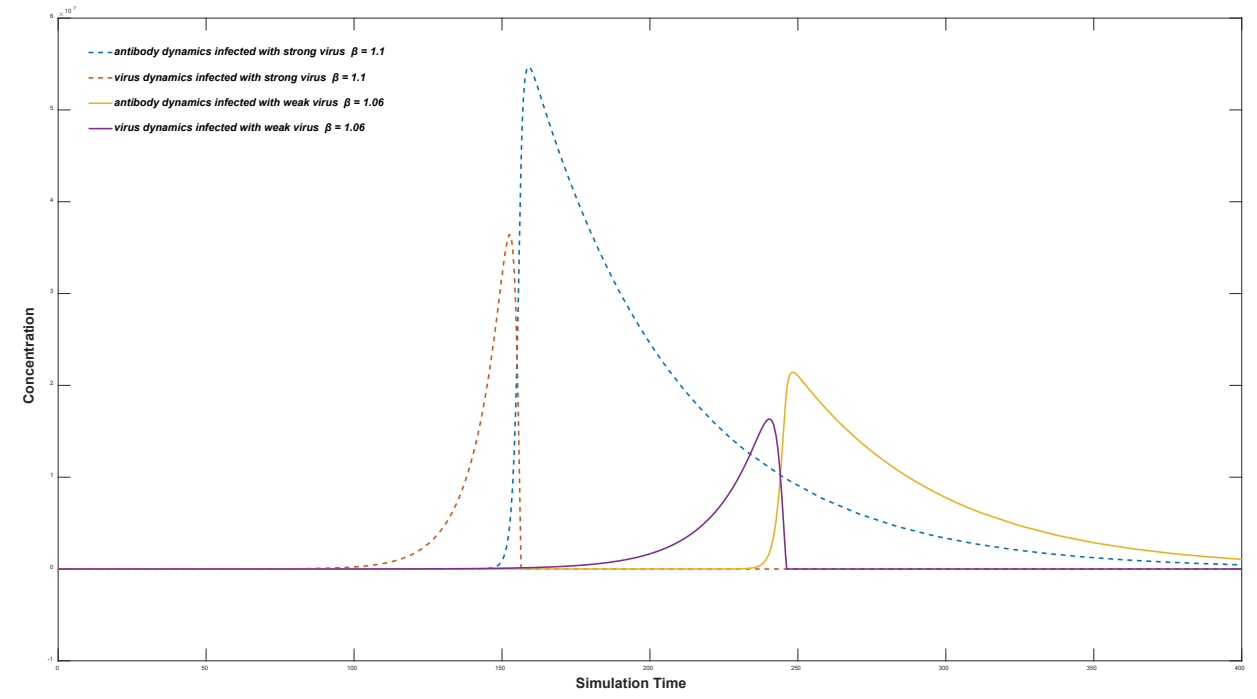

Figure 3: Different immune behaviors toward variants with different replication activities. The parameter sets we used are $\mathrm{x}(0)=0, \mathrm{y}(0)=100, \mathrm{z}(0)=10, \mathrm{q}(0)=1000000, \mathrm{k} 1=1 \mathrm{e}-5, \mathrm{k} 2=1 \mathrm{e}-14, \mathrm{k} 3=$ $1 \mathrm{e}-7, \mathrm{k} 4=1 \mathrm{e}-14 . \alpha=0.98, \quad \beta=1.05$ or 1.1 .

It can be noticed from figure 3 that under equal initial viral invasion dose, the infection caused by a virus with weak replication has a longer latency phage significantly, and its peak viral load is significantly lower than that of a strong replication activity virus. This weak virus infection would induce significantly lower antibody production than that produced by a strong replication activity virus, but the infection cycle is significantly longer than that of a strong replication activity virus.

\section{How the immune system screens for highly binding antibodies.}

Our immune system can automatically select those suitable binding antibodies and selectively proliferate them. How to simulate the antibody screening process by mathematical modeling remains a challenge for computational biology researchers. Our model shows that there is a significant correlation between the proliferation potential and its binding kinetics. Antibodies with fast binding rates can gain rapid proliferation, while those with low binding rates have significantly weaker proliferation potential. Thus, although antibodies with different binding capacities are all triggered to proliferate during infection, the antibodies with fast binding rates can grow faster and dominate in the composition of the final antibodies. In general, antibodies with solid forward binding kinetics have a more robust relative binding affinity, but this is not an absolute relationship. Binding affinity is the ratio of forwarding binding to the rate of reverse dissociation. Therefore, the vast majority of infected individuals would have antibodies with excellent binding kinetics after infection. However, those antibodies display individual differences in binding affinity, with some antibodies having relatively strong binding affinity. 
The dominant factor affecting the efficiency of antibodies is not their absolute binding affinity Kd but the reaction constant of the binding process, which represents $\mathrm{k} 1$.

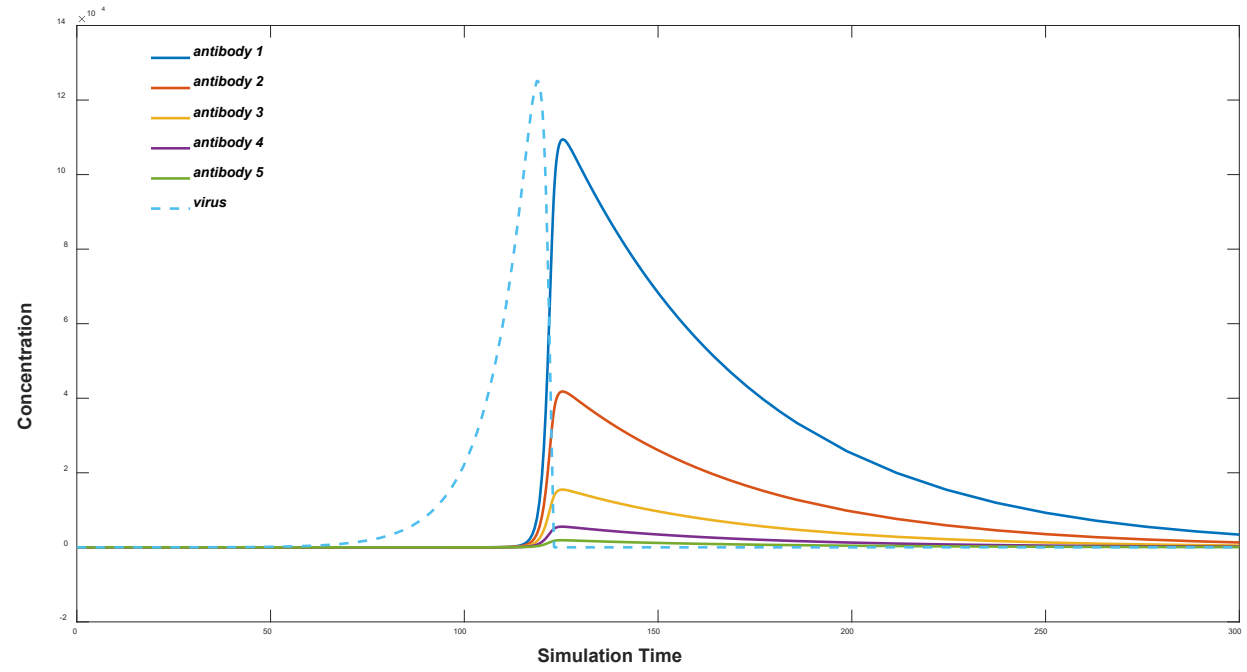

Figure 4: Dynamics of different antibody with different kinetics attributes

The parameter sets we used are

$\mathrm{x}(0)=0, \mathrm{y}(0)=1, \mathrm{z}(0)=1, \mathrm{w}=1, \mathrm{k} 1=1 \mathrm{e}-5, \mathrm{k} 2=1 \mathrm{e}-14, \alpha=0.98, \quad \beta=1.1$ for antibody $1 ;$

$\mathrm{x}(0)=0, \mathrm{y}(0)=1, \mathrm{z}(0)=1, \mathrm{w}=1, \mathrm{k} 1=9 \mathrm{e}-6, \mathrm{k} 2=9 \mathrm{e}-15, \alpha=0.98, \quad \beta=1.1$ for antibody 2 ;

$\mathrm{x}(0)=0, \mathrm{y}(0)=1, \mathrm{z}(0)=1, \mathrm{w}=1, \mathrm{k} 1=8 \mathrm{e}-6, \mathrm{k} 2=8 \mathrm{e}-15, \alpha=0.98, \quad \beta=1.1$ for antibody 3 ;

$\mathrm{x}(0)=0, \mathrm{y}(0)=1, \mathrm{z}(0)=1, \mathrm{w}=1, \mathrm{k} 1=7 \mathrm{e}-6, \mathrm{k} 2=7 \mathrm{e}-15, \alpha=0.98, \quad \beta=1.1$ for antibody 4 ;

$\mathrm{x}(0)=0, \mathrm{y}(0)=1, \mathrm{z}(0)=1, \mathrm{w}=1, \mathrm{k} 1=6 \mathrm{e}-6, \mathrm{k} 2=6 \mathrm{e}-15, \alpha=0.98, \quad \beta=1.1$ for antibody 5 ;

As shown in Figure 4, an antibody with a fast binding rate, those that bind quickly, proliferate significantly during infection. This selection will further result in a higher proportion of the overall antibody. Although these five antibodies have the same binding affinity with $\mathrm{Kd}=1 \mathrm{e}-9$, their proliferation varies because they bind at different rates, and the antibody with the faster binding rate will gradually dominate. This example illustrates that the driving force in antibody reproduction after infection is its binding speed, not binding affinity.

4 High concentrations of weakly binding antibodies can provide effective protection.

For COVID-19 infection, we often find it interesting that although we do not understand the mechanism, it is statistically proven that vaccination with other vaccines such as the influenza vaccine can also provide some degree of protection against COVID-19[23-26]. Here we use our model to explain this phenomenon. 


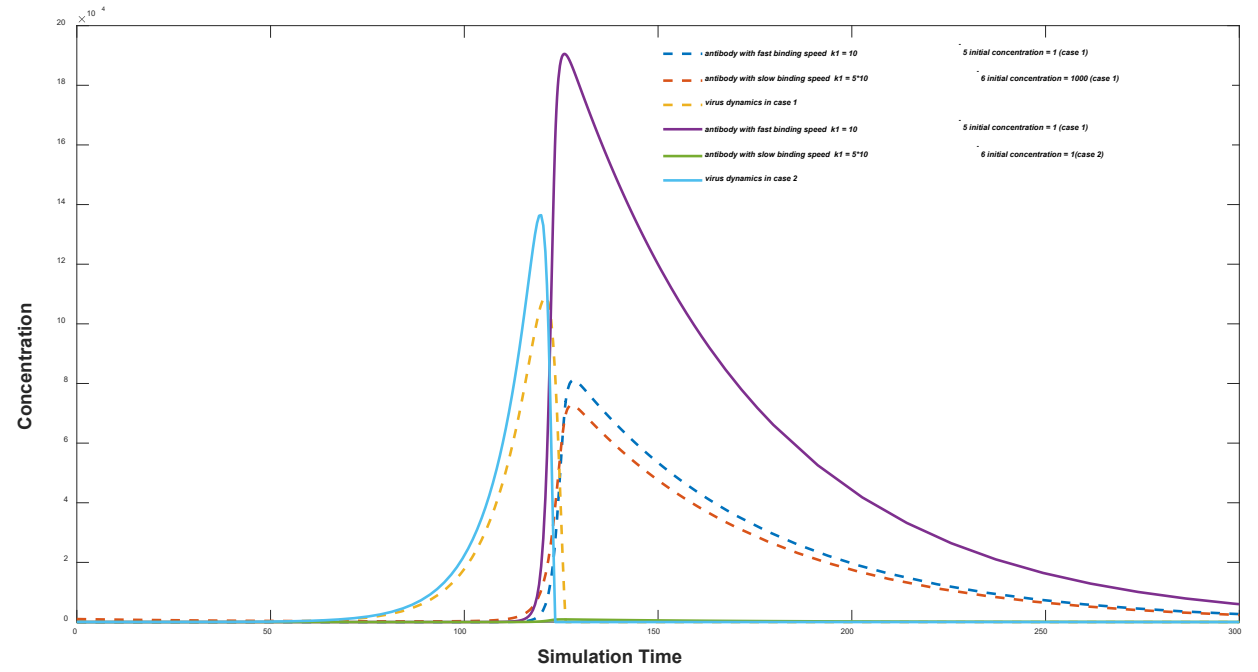

Figure 5: High concentrations of weakly binding antibodies can provide effective protection

A plot of the inhibitory capacity of a specific concentration of weakly binding antibodies against the virus (two antibodies, one strong and one weak, but the weak antibody has a higher initial concentration 1000 , antibody 1 strong binding, but an initial concentration of 1 )

The parameter sets we used are

$\mathrm{X} 1(0)=0, \mathrm{X} 2(0)=0, \mathrm{y} 1(0)=1, \mathrm{y} 2(0)=1000, \mathrm{z}(0)=1, \mathrm{w} 1=1, \mathrm{w} 2=1, \operatorname{Kon}_{1}=1 \mathrm{e}-5, \operatorname{Koff}_{1}=1 \mathrm{e}-14$, Kon $_{2}=5 \mathrm{e}-6$, Koff $_{2}=1 \mathrm{e}-14, \quad \alpha=0.98, \quad \beta=1.1$ for case 1 ;

$\mathrm{X} 1(0)=0, \mathrm{X} 2(0)=0, \mathrm{y} 1(0)=1, \mathrm{y} 2(0)=1, \mathrm{z}(0)=1, \mathrm{w} 1=1, \mathrm{w} 2=1$, Kon $_{1}=1 \mathrm{e}-5, K_{\text {off }}=1 \mathrm{e}-14$, Kon $_{2}$

$=5 \mathrm{e}-6, \operatorname{Koff}_{2}=1 \mathrm{e}-14, \quad \alpha=0.98, \quad \beta=1.1$ for case 2 ;

Figure 5 illustrates that a particular concentration of weakly binding antibodies can produce an inhibitory capacity against the virus. A decrease in peak viral load in case 1 compared to case 2 can be notified in figure 5. Figure 5 also illustrates that a particular concentration of weakly binding antibodies can inhibit the proliferation of strongly binding efficient antibodies in the organism. For both cases, the analysis in the next section will show that their ability to resist secondary infection is also different.

This example could also suggest that vaccinated people will always have better protection against initial infection than non-vaccinated people, regardless of the mutation of the virus. However, we should also be cautious about the disadvantages brought by vaccination with other types of vaccines.

We aim to stimulate the production of high levels of firmly bound active antibodies by vaccination, but a large amount of weakly bound active antibodies primordially present in the body interferes with and disrupts this process. Therefore, vaccination will result in significant efficiency differences, with some individuals producing large amounts of fast binding active antibodies. On the contrary, individuals with antibody interference would not readily produce large amounts of highly active antibodies, thus showing a tremendous difference in protection efficiency. The effect of antibody interference on vaccine efficacy is complicated and, through simulation, this effect can be quantitively evaluated. The simulation results indicate antibody interferences might be greatly enhanced for people whom some other vaccines have been vaccinated. Therefore, people with pre-vaccination with other vaccines might be limited in triggering fast and efficient antibodies against vaccination targets. This could help explain why some vaccines will lead to a higher overall mortality rate in some instances. Keeping a relatively high antibody level for a specific pathogen such as COVID-19 will shelter more people from infection. However, it might also play a side effect on decreasing the efficiency of other vaccines. Therefore, there is a significant concern about COVID-19 vaccination with juveniles. Even for adults, repeated booster vaccination might significantly decrease their immunity against other pathogens.

\section{Calculation of the protection time brought by natural infection}




\subsection{A simple calculation of the antibody protection time}

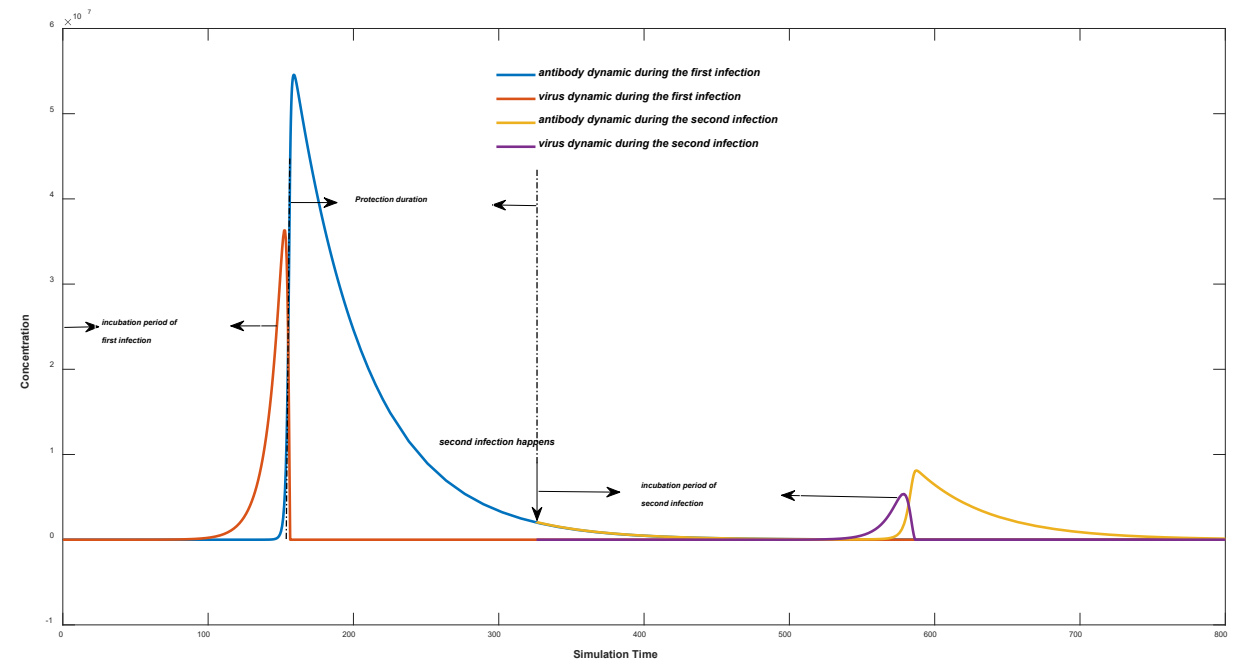

Figure 6: an illustration of protection time calculation

The parameter sets we used are

$\mathrm{x}(0)=0, \mathrm{y}(0)=100, \mathrm{z}(0)=10, \mathrm{q}(0)=10000, \quad \mathrm{k} 1=1 \mathrm{e}-7, \mathrm{k} 2=1 \mathrm{e}-14, \mathrm{k} 3=1 \mathrm{e}-8, \mathrm{k} 4=1 \mathrm{e}-14, \alpha=0.98$,

$\beta=1.1$.

The second infection time is marked in figure 6. The second infection would start around 326-th time unit. Before that time point, infection will not cause any virus proliferation. Therefore, the protection time can be roughly estimated, starting from 166th to 326th. From figure 6, we can also notice that maximal virus load in the second infection that happened at the 326th time point is significantly lower than the first infection. This means that although people will be infected at the 326th time point, it still displays a protective effect since the peak virus load is much smaller than the initial infection. However, the protective effect will decrease as time increases. This situation will be shown in Figure 7 in the following part.

5.2 Factors affecting the duration of antibody protection: concentration of environmental antigenlike substance, viral replication capacity and antibody binding kinetics.

As shown in equation (17), as long as $\left(1-\mathrm{q}^{*} \mathrm{k} 3\right)^{*} \beta>1$, the virus will reproduce itself. Therefore, the virus replication capacity and the environmental antigen-like substances directly influence the protection time of a particular antibody. A lifelong protective effect can happen when a virus has a weak replication capacity, a high concentration of environmental antigen-like substances, and a very fast binding antibody with an enormous $\mathrm{k} 1$ value. Good examples of this category are Variola virus, tetanus bacillus, and so on. However, other viruses such as influenza or sars-cov-2 may not be able to trigger a superlong protection time. Meanwhile, for influenza or sars-cov-2 infections, there is a considerable variation in the protective time after natural infection or vaccination. This variation is mainly contributed to the difference in their corresponding antibody kinetics.

As we demonstrated above, the binding kinetic constant $\mathrm{k} 1$ is much more important than the binding affinity $\mathrm{Kd}$. Here below, we illustrate how $\mathrm{k} 1$ influences protection time in different infection cases. 


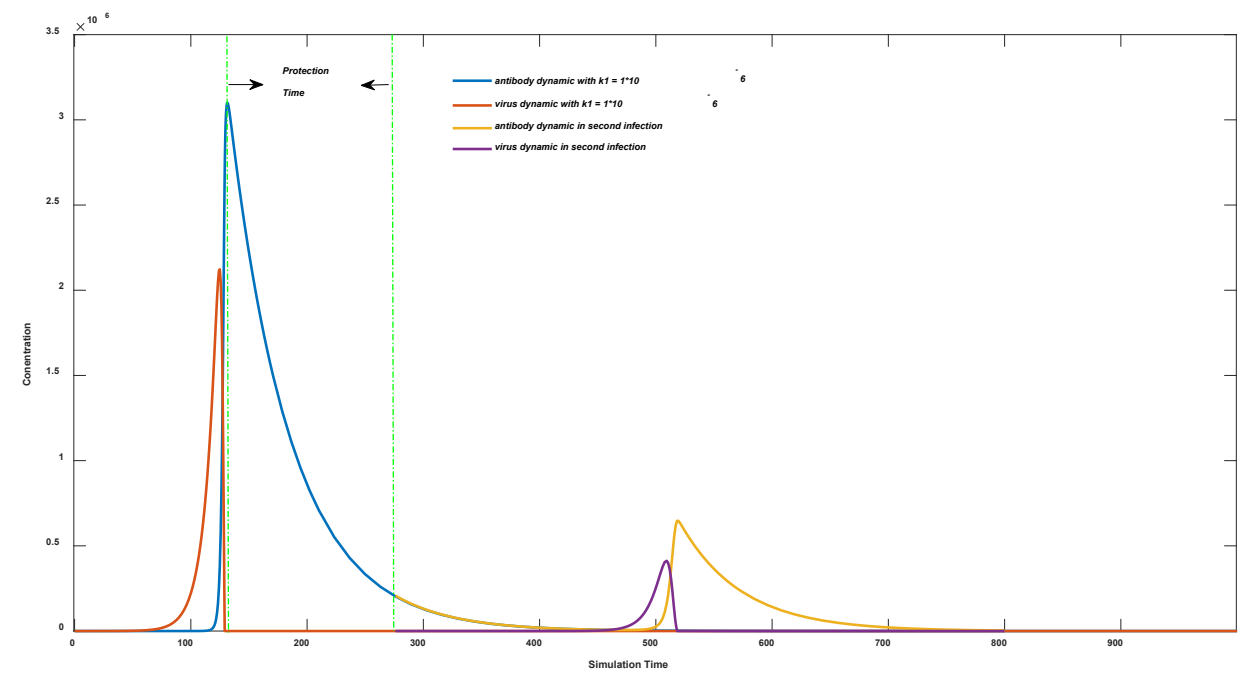

Figure 7A: Protection time calculation when the antibody has a binding kinetics k1 $=1 \mathrm{e}-6$. The parameter sets we used are

$\mathrm{x}(0)=0, \mathrm{y}(0)=100, \mathrm{z}(0)=10, \mathrm{q}(0)=1 \mathrm{e} 6, \quad \mathrm{k} 1=1 \mathrm{e}-6, \mathrm{k} 2=1 \mathrm{e}-14, \mathrm{k} 3=1 \mathrm{e}-9, \mathrm{k} 4=1 \mathrm{e}-14, \alpha=0.98, \quad \beta$ $=1.1$.

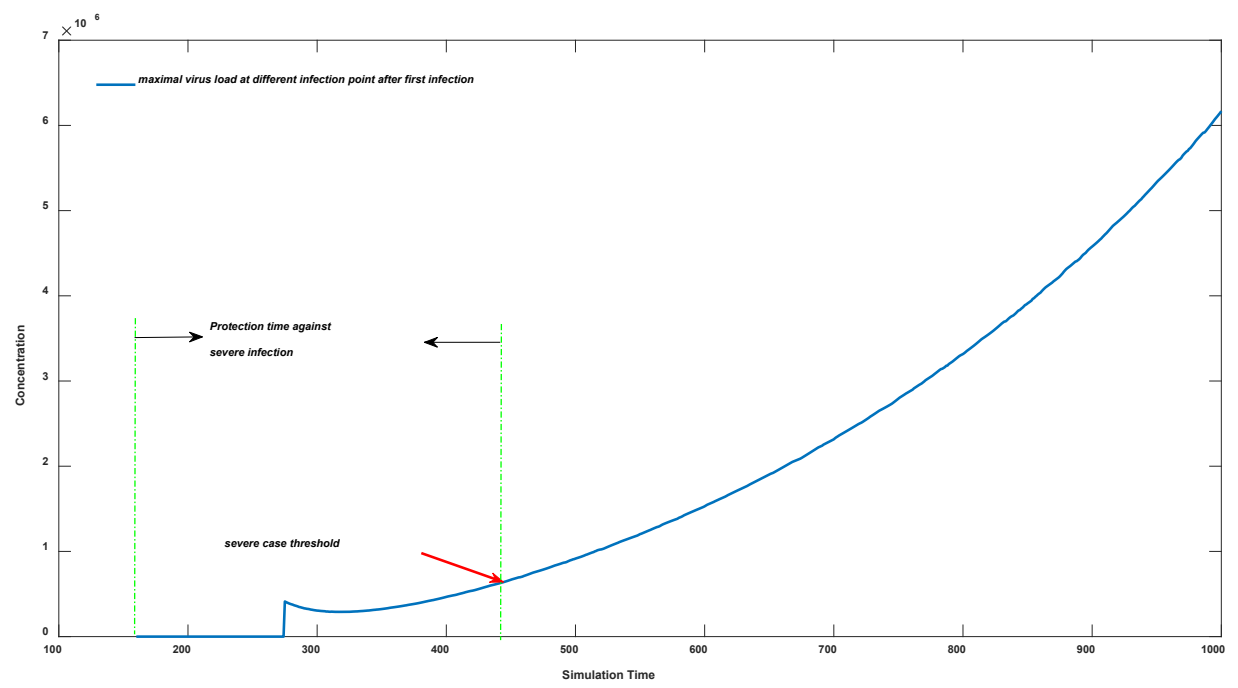

Figure 7B: Maximal virus load at different infection point when the antibody has a binding kinetics $\mathbf{k 1}=\mathbf{1 e - 6}$.

The second infection time is marked in figure 7A. The second infection would start around the 275th time unit. Before that time point, infection will not cause any virus proliferation. Therefore, the protection time can be roughly estimated, which is started from 125th to 275 th-time unit. From figure $7 \mathrm{~B}$, we can also notice that maximal virus load in the second infection will slightly decrease from the 275th-time unit to the 330th-time unit but will gradually increase after passing the 330th-time unit. If we define a threshold as a severe case, one that we arbitrarily set as $6.4 \mathrm{e} 5$ marked by the red arrow in figure $7 \mathrm{~B}$, then we can estimate the protection time against severe infection, which is the time interval between those two green lines. 


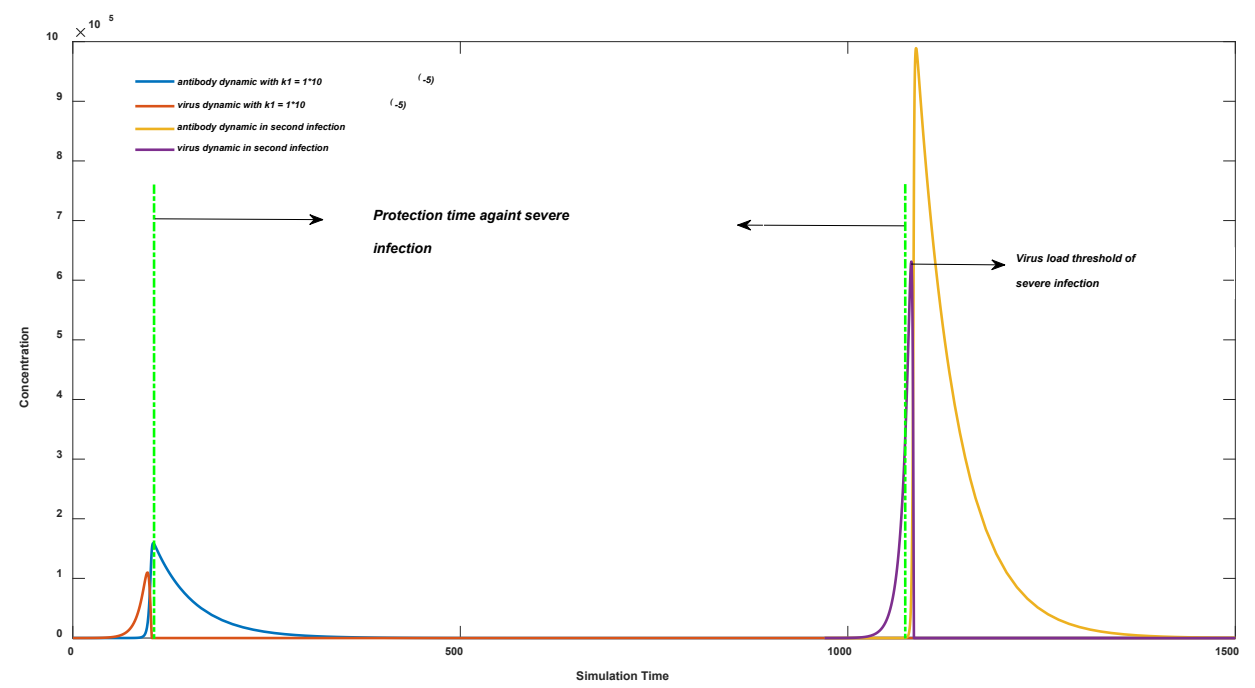

Figure 8A: Protection time calculation when the antibody has a binding kinetics k1 $=1 \mathrm{e}-5$. The parameter sets we used are

$\mathrm{x}(0)=0, \mathrm{y}(0)=100, \mathrm{z}(0)=10, \mathrm{q}(0)=1 \mathrm{e} 6, \quad \mathrm{k} 1=1 \mathrm{e}-5, \mathrm{k} 2=1 \mathrm{e}-14, \mathrm{k} 3=1 \mathrm{e}-9, \mathrm{k} 4=1 \mathrm{e}-14, \alpha=0.98, \quad \beta$ $=1.1$.

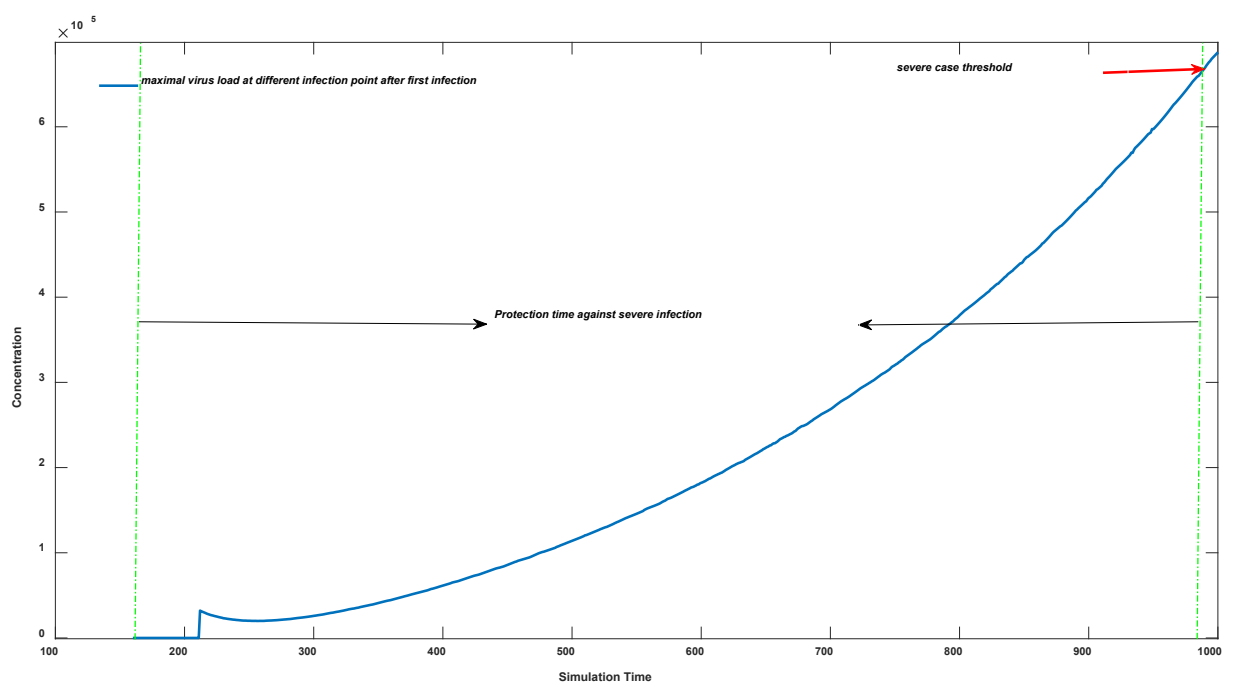

Figure 8B: Maximal virus load at different infection point when the antibody has a binding kinetics k1 = 1e-5.

As shown in figure 8, the severe infection would happen after the 975th time unit with the same severe threshold as in figure 7. Therefore, the protection time can be roughly estimated, which is started from 125th to 975 th-time unit. This protection phase is much longer compared to the case when $\mathrm{k} 1=1 \mathrm{e}-5$. This example demonstrates that an excellent binding antibody will protect people longer than the weak one. An exciting discovery from our modeling is that people generating suitable antibodies with fast binding kinetics rarely display significant symptoms in the first infection. This situation can be reflected in the smaller virus peak loading amount in the first infection in figure $8 \mathrm{a}$ compared to figure $7 \mathrm{a}$. Although experiment researchers seek antibodies with super excellent binding affinity, we show that we should better seek antibodies with super-fast binding kinetics, and those fast-binding antibodies typically existed with patients without intense infection symptoms. Repeated vaccination has the effect of increasing the overall antibody level for a short period but does not reshape the composition of the 
antibodies, i.e., it does not significantly alter the binding activity of the antibodies. Therefore, in the long term, improving antibody binding activity against a strongly replicating virus is the key to improving vaccine protection, and repeated vaccinations will only protect for a relatively short time. The heterogeneity of the human body causes significant differences in the binding activity of antibodies produced by different individuals to the same viral infection or vaccination, thus causing significant differences in protection duration in different individuals. Therefore, we may need to change the vaccination strategy, and in the future, vaccination should seek to produce high levels of antibodies and be able to induce targeted production of antibodies with high binding activity. The current use of gene editing to induce the production of specific antibodies in a targeted manner is a good direction [27-29].

\section{3 parameter estimation}

The kinetic parameters $\mathrm{k} 1$ values for antibody-antigen binding and kinetic parameters $\mathrm{k} 3$ for environmental antigen-like substances can be obtained with certainty through parameter fitting.

In order to better calculate the protective cycle of antibodies, we need to determine these parameters in advance, where the initial number of antibodies and pathogens, as well as the content of antigen-like substances in the environment, do not have a significant effect on their decay. In contrast, the parameter $\alpha$ can be treated as a fixed value since we consider that all B or T cells have the same half-life, independent of the type of antibody they express. Whereas $\beta$ indicates the replication rate of the virus and is related to the virus itself, this parameter can be obtained by calculating the rate of viral proliferation in the early stages, when the amount of antibodies are relatively small. Of the other four parameters, we defined k2 and k4 as smaller numbers because they are small and have fragile effects on antibody and virus kinetics changes by parameter sensitivity analysis. The key to parameter fitting is to obtain values for the two positively bound kinetic parameters, $\mathrm{k} 1$ and $\mathrm{k} 3$.

We show that when $\mathrm{k} 1$ and $\mathrm{k} 3$ are within the normal range of reaction dynamics, those two parameters can be uniquely solved by a global search method.

We take $\mathrm{k} 2=\mathrm{k} 4=1 \mathrm{e}-14 ; \alpha=0.98 ; \beta=1.1 ; \mathrm{y}(0)=100 ; \mathrm{z}(0)=10 ; \mathrm{q}(0)=1 \mathrm{e} 6$.

When $\mathrm{k} 1$ is taken from $1 \mathrm{e}-3$ to $1 \mathrm{e}-7$, and $\mathrm{k} 3$ is taken from $1 \mathrm{e}-7$ to $1 \mathrm{e}-11$, a global search uniquely estimates $\mathrm{k} 1$ and $\mathrm{k} 3$.

For example, when $\mathrm{k} 1=1 \mathrm{e}-5$ and $\mathrm{k} 3=2 \mathrm{e}-8$, we calculate the kinetic profiles of their antibodies and viruses through a system of ordinary differential equations displayed in Figure 9. By selecting these points as experimental data at parameter estimation, $\mathrm{k} 1$ and $\mathrm{k} 3 \mathrm{can}$ be uniquely recovered by parameter estimation. In another way, we can determine the values of $\mathrm{k} 1$ and $\mathrm{k} 3$ by fitting the experimental data. The details of the global search are described in the methods section. After estimating those parameters, we can further calculate the protection time according to the approach illustrated in figure 7 and 8 . 


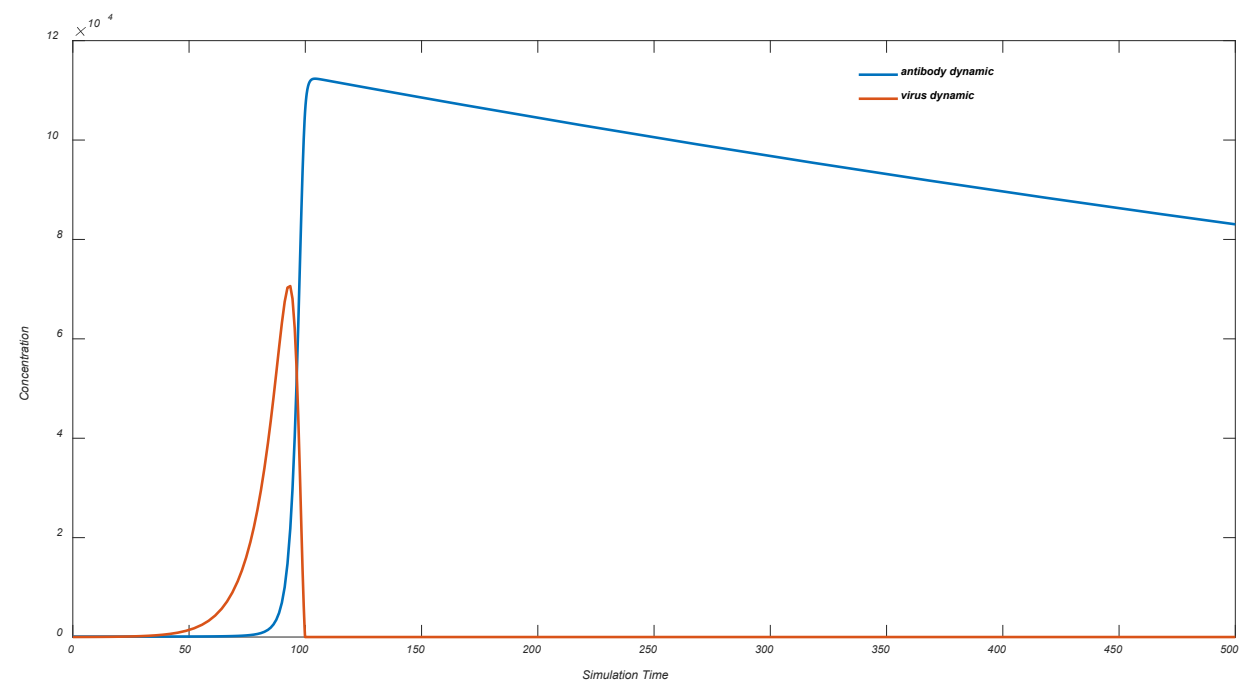

Figure 9 : antibody and virus dynamics when $k 1=1 e-5, k 3=2 e-8$.

\subsection{Recovered COVID-19 Patients with Retest Positive for SARS-CoV-2 RNA}

In COVID-19 infections, we will note the presence of reinfections by patients themselves, although it is not very common [30,31]. The self-reinfection phenomenon may lead to a second epidemic outbreak, making it more challenging to prevent and control the epidemic. The scenario of self-reinfection is a frequent phenomenon in virus infection. For influenza infection, we often hear of the phenomenon of self-reinfection or infection recovery, which means that patients who have just recovered from a cold may become infected again. It is very typical when HBV or HCV infection occurs. This reinfection is not a secondary infection caused by the outside world but the result of self-infection. Our model can explain this phenomenon. The patient will experience reinfection under certain parameter sets, as shown in

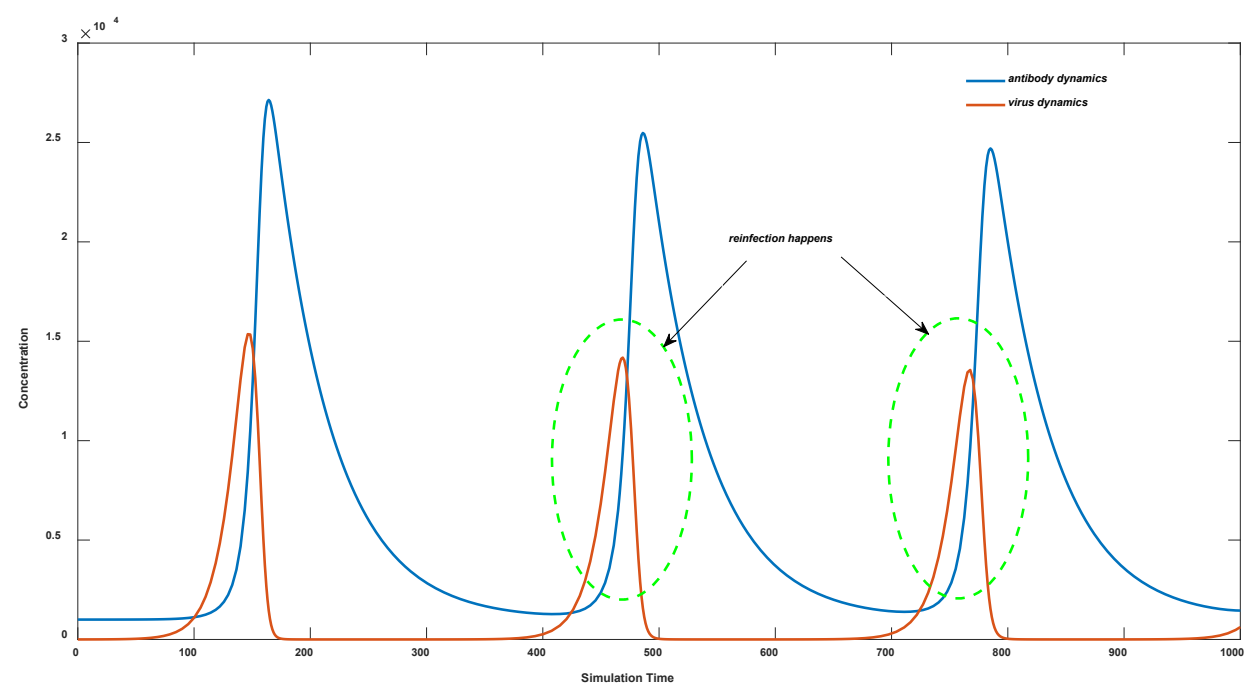

Figure 10: self-reinfection scenario

The parameter sets we used are $\mathrm{x}(0)=0, \mathrm{y}(0)=1000, \mathrm{z}(0)=1, \mathrm{w}=1000, \quad \mathrm{k} 1=1 \mathrm{e}-5, \mathrm{k} 2=1 \mathrm{e}-14, \alpha=0.98, \quad \beta=1.08$.

This example above shows that when a certain number of fast-binding antibodies are present in the body, the probability of a repeated positive nucleic test will be greatly increased. However, the peak viral load 
of the initial infection is small, and the symptoms triggered by the initial infection are relatively mild. Due to the interaction kinetics between the viruses and the antibodies, the number of antibodies induced by the initial infection is insufficient. Therefore, it is impossible to eliminate the virus from the body, resulting in a low remaining virus level. When the antibody level decreases later, the virus will regain the opportunity to proliferate, and the patient may display a positive result again in the future nucleic acid test. However, the infections that in those cases are generally less symptomatic or asymptomatic. A more extreme case is a long-term chronic infection, such as the appearance of a long-positive patient.

\subsection{Mechanisms of chronic infection}

When antibodies with fast binding activity are present in the body, the peak load of pathogenic substances will not be too high because of the fast-binding activity of the antibodies, which effectively inhibits the proliferation of large amounts of pathogenic substances. However, a side effect of that will lead to the situation that they cannot sustainably stimulate the proliferation of antibodies in large amounts, and therefore the level of antibodies cannot reach a very high level. In this case, the kinetic characteristics of both sides might allow the two to reach a state of equilibrium at a lower level, thus presenting a chronic inflammatory character which is shown in figure 11 below.

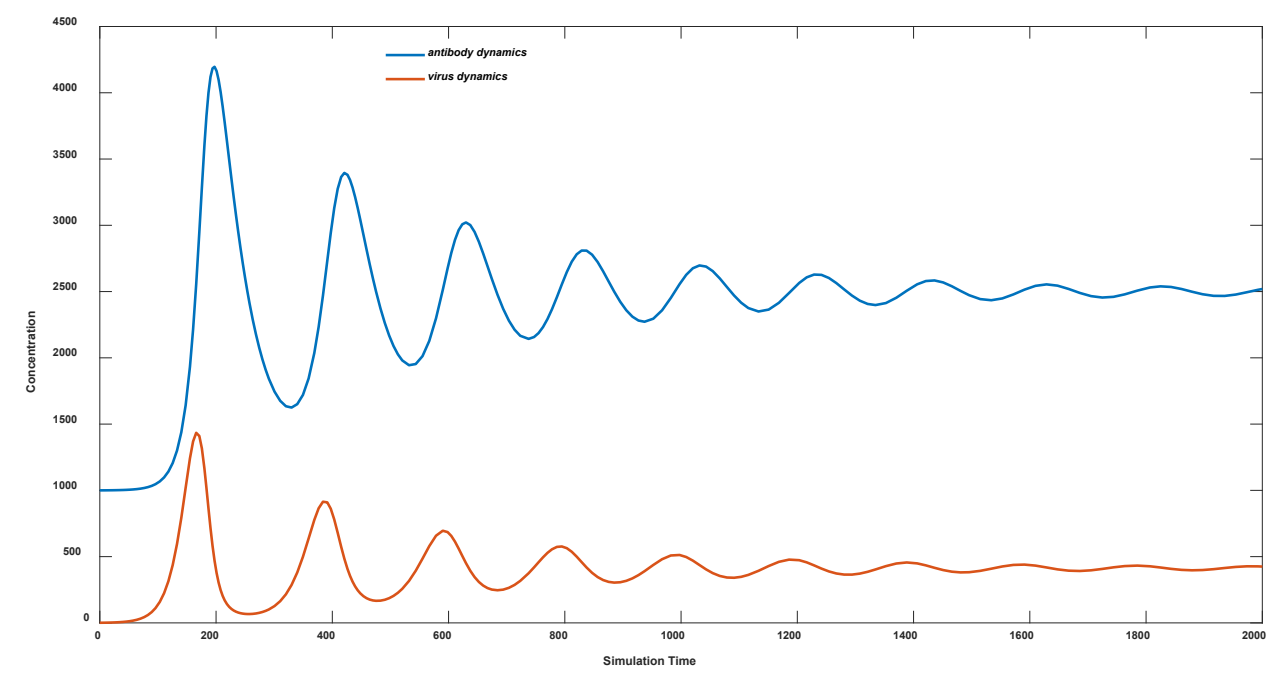

\section{Figure 11: scenario of chronic infection}

The parameter sets we used are

$\mathrm{x}(0)=0, \mathrm{y}(0)=1000, \mathrm{z}(0)=1, \mathrm{w}=1000, \quad \mathrm{k} 1=3 \mathrm{e}-5, \mathrm{k} 2=1 \mathrm{e}-14, \alpha=0.98, \quad \beta=1.08$.

Chronic inflammation can occur due to two factors. Pathogenic microorganisms cause one, and allergic substances in the environment cause the second. For chronic infections caused by pathogenic microorganisms, in addition to the use of anti-pathogen drugs, we might try a short-term boost in antibody levels to accomplish a complete clearance of pathogenic microorganisms. From clinical experience, we had experienced many instances where chronic infections had permanently disappeared or significantly diminished when they turned out to be acute infections. As the acute infection fades away, chronic infection will disappear or become invisible for a long time. We normally treat chronic inflammation caused by environmental factors by shielding allergen-like substances for a certain period. The patient's antibody levels would lower, thereby attenuating or eliminating the immune response, mainly about decreasing the specific antibody level.

6 Kinetic models of viruses and antibodies can reflect the heterogeneity in viral replication capacity

Based on our model, we further investigated the dosimetric characteristics of infection. To our surprise, the most important features affecting the severity of the infection are two parameters, specifically speaking, the viral replication activity $\beta$ and antibody binding constant $\mathrm{k} 1$. In contrast, the number of viral invasions had a weak effect on the final viral load but significantly affected the time to peak, which is mainly denoted as the incubation period. This could also explain why the virus's incubation period is 
significantly shorter for delta mutant strains whose concentrations in the respiratory system are remarkably higher than the original strain [32].

However, we have difficulty explaining a significant relationship between virus invading dose and symptom severity. Low-dose viral invasion tends to result in a lower proportion of severe cases/overall infection cases, while high-dose invasion tends to result in a higher proportion of severe cases /overall infection cases. This phenomenon is more clearly demonstrated in masks, where there is evidence that masks reduce the risk of infection and significantly reduce the severity of symptoms [33-36]. We can only explain this phenomenon by introducing viral heterogeneity, specifically, that not every virus in every environment has the same replication capacity. However, their genetic sequences are almost indistinguishable (since they all originate from the same infectious individual). This genome heterogeneity could only derive from differences in the length of the UTR (Untranslated region). This heterogeneity in replication capacity results in dosimetric differences in infectivity. As a simple example, suppose the environment consists of $\mathrm{n}$ viruses, $10 \%$ of which are high replication activity and $90 \%$ of which are low replication activity. When a single virus invades the body, there is only a $10 \%$ probability that the infected person will exhibit a high peak viral load, i.e., a severe case of disease. Nevertheless, when $\mathrm{n}$ viruses enter the cell, the infected person has a probability of 1-0.9n of exhibiting severe disease, and the larger the number of invading viruses $n$, the greater the probability of severe disease. This phenomenon strongly validates our UTR decay theory [37]. This is because only the attenuation of the UTR region could lead to significant differences in replication activity exhibited by viruses released into the environment from the same host.

\section{Conclusion}

Engels once said that "the true perfection of any science lies in the extensive use of mathematical tools." Mathematician Russell also said that "no subject can be a science without the introduction of mathematics." In immunology, the proper use of mathematical models is also essential, as they can help us explain the dynamics of the immune process quantitatively. We believe that mathematical modeling should be based not on simple function selection and data fitting but a mechanism supported by concrete physical meaning. It is based on this idea that we propose the antibody kinetic hypothesis and elaborate its theoretical supports. Based on this theory, we can better explain several interesting and mysterious phenomena mentioned in the introduction.

First: How are memory cells maintained?

According to our theory, we believe that memory cells are maintained through environmental antigenlike substances. Through a process of kinetic simulation and mathematical derivation, we can demonstrate that in most cases, environmental antigen-like substances cannot directly stimulate antibody proliferation until the organism is exposed to fundamental pathogenic antigenic substances capable of proliferation. In most cases, antigen-like substances do not induce the process of antibody proliferation and screening but significantly reduce the rate of decay of the induced antibody production. Thus, the maintenance of memory cells is closely linked to antigen-like substances in the environment, and some memory cells expressing specific antibodies can be maintained in the human body for decades. The longevity of memory cells is not because those memory cells have extremely long half-lives, but because they can be subjected to constant stimulation by antigen-like substances in the environment, resulting in partial proliferation, which will compensate for the decay effects, and thus can be maintained for a considerable period. In rare cases, when the binding activity of environmental antigenic substances to specific antibodies is super-strong, or when the concentration of environmental antigenic substances is too high, those non-pathogenic antigenic substances in the environment can also induce the proliferation of antibodies and produce some allergic reactions, such as allergic rhinitis, etc.

Second: How does our immune system screen for antibodies that have a strong binding affinity?

According to our model, antibodies with fast binding kinetics proliferated substantially. In general, a positive correlation is shown between binding affinity and binding kinetics, but this is not an absolute relationship. Thus, not all recovered individuals are screened for antibodies with particularly excellent binding affinity. However, the immune system preferentially screens for antibodies with fast binding rates, a kinetic parameter that is more important than absolute binding affinity, especially at the level of 
inhibition of pathogenic microorganisms. It is not surprising that dissociation constants determined experimentally are usually significantly weaker than values derived from structure-based theoretical calculations. This does not indicate the inaccuracy of the energy functions used in the theoretical calculations. The binding energies calculated from structure-based theoretical calculations can be further used to derive dissociation constant. This dissociation constant is the dissociation coefficient at absolute equilibrium conditions. However, due to the kinetic nature of the reactions, such equilibria cannot be reached in experimentally accessible times, so experimentally determined dissociation constants are usually weaker than the values derived from structure-based theoretical calculations.

Third: Why do people who vaccinated influenza or other vaccines have a lower mortality rate from COVID-19?

An interesting phenomenon during sars-cov-2 pandemic is that the mortality rate of COVID-19 is significantly lower in people who have received influenza, or other vaccines, than in those who have not [23-26]. Our antibody kinetic simulations can explain this phenomenon well; vaccination with other vaccines has the potential to elevate the level of nonspecific binding antibodies, i.e., weak binding rate antibodies. Although the presence of such weakly binding antibodies inhibits the proliferation of strongly binding antibodies in vivo, they can significantly inhibit virus proliferation and reduce peak viral load compared to controls, thus exhibiting lower mortality and severe disease rates. However, this situation is not without its drawbacks. As mentioned above, high concentrations of weakly binding antibodies inhibit the proliferation of strongly binding antibodies in vivo. Therefore, although statistical data is temporarily lacking to support this prediction for those vaccinated with influenza and other vaccines, we speculate that their subsequent vaccination with the COVID-19 vaccine may be significantly less protective than those not vaccinated with influenza.

Fourth: How could we effectively calculate the protection duration of a specific antibody?

Since statistical data demonstrate that the protection of the COVID-19 vaccine declines over time, more and more attention is paid to this topic. If there are statistical data for a population, which mainly include dynamics of viral and antibody changes over time, we can estimate the antibody kinetic parameters of this population through a data fitting process. Those parameters could also include the initial concentration of environmental antigen-like substances and other initial factors. We can further calculate the protection time of the vaccine or natural infection based on those parameters. A more accurate method is personalized prediction. If data for a specific individual is available, we can also fit individual parameters and thus personalize an individual's antibody protection cycle prediction. This personalized protection time can vary widely, with some people showing extraordinarily long protection cycles and others relatively short. Our theory can also explain significant differences in their corresponding antibody protection time for different pathogenic microorganisms. In general, the variability in immune protection time is mainly related to several factors. The first is that the replicative activity of the virus with strong replicative capacity has shorter antibody protection times. The second is the T-cell immunogenicity of the virus. Viruses with strong T-cell immunogenicity can quickly proliferation memory cells and exhibit long protection or even lifelong protection. The third is the differences in the antibody binding kinetics toward target pathogen antigen. Differences in antibody attributes will also lead to differences in the binding kinetics of the corresponding antigen-like substances in the environment. Therefore, this will exhibit different decay rates, and protection time will be correspondingly longer for those with slower decay rates.

Fifth: Why are there cases of self-reinfection?

In covid-19 infections, the phenomenon of retest positives without infections from other people is frequently reported [30-31]. This dramatically increases the difficulty of epidemic prevention and control, and it is difficult to explain this phenomenon without a mathematical simulation. Our model shows that individuals will exhibit self-reinfection for specific combinations of parameters that include antibody kinetic, concentrations of environmental antigen-like substances, and viral replication coefficients. When a specific concentration of antibodies with fast binding kinetics is present in the body, or when viral replication is weak, the initial infection will show asymptomatic or mild symptoms, the probability of future self-reinfection is greatly increased. An extreme case is a long-positive phenomenon in which the individual continues to show positive nucleic acid results. Generally, the virus in a long-positive patient is fragile in its ability to proliferate, and the virus usually is less virulent. 
Sixth: How are chronic infections triggered?

Chronic inflammation is a significant problem that plagues human health, and chronic inflammation can be divided into two categories, chronic infections caused by pathogenic microorganisms and chronic allergic inflammation due to environmental factors. The mechanism by which chronic infections occur could also be well demonstrated by our model, which states that, under a specific combination of parameters, pathogenic microorganisms can reach a steady state with their corresponding antibodies at a relatively low level, thus exhibiting a long-term chronic inflammation.

Seventh: Why do vaccinations show considerable differences in protection?

According to our antibody kinetic model, the most crucial reason for the differences in protection produced after vaccination, apart from viral mutations, lies in the differences in the attributes of the antibodies induced by the vaccine. The first factor is the presence of antibody interference, that is, the presence of different concentrations of interfering, or weakly binding, active antibodies in different vaccinees. Large amounts of interfering antibodies can inhibit the production of high concentrations of fast binding antibodies in the vaccine, causing a decrease in protection efficiency and protection period. Another factor is that at the same level of antibody interference, different individuals could also display heterogeneity in producing antibodies with different binding kinetic attributes, providing different degrees of protection against secondary infections. Those antibodies could also have different kinetic behaviors with their corresponding environmental antigenic substances and therefore have various decay rates. Antibodies with slower decay rates will provide more vital protection and longer protection time.

Eighth: How can we improve the protective efficiency and duration of vaccines?

According to our model, persistent high-dose exposure to antigenic substances can increase antibody levels in a short period, but this is not the most effective method, and repeated booster shots are less likely to change the antibody composition. The best way to provide prolonged protection against the same virus, such as COVID-19, is to induce the production of an antibody with superb fast binding activity in a targeted manner. Although this technology is still in its infancy, many researchers have tried to open this direction through gene-editing techniques [27-29]. Another concern with the constant booster shots is that this approach is highly likely to diminish the effectiveness of other vaccines, including future vaccines against massively mutated strains of sars-cov- 2 . Because this approach maintains the level of specific antibodies in the body at a relatively high level, it will inhibit the production of specific antibodies with fast binding activity against future mutant strains, such as a future covid-19 vaccine for mutant strains. Increasing the dose of the vaccine could also produce higher antibody levels, but this would pose a greater risk of vaccine side effects. Therefore, it should be recognized that while there is a correlation between the protection duration of a vaccine and its peak antibody levels, this correlation is not strong. Simply pursuing a higher peak antibody level is not the most effective means of extending the protection duration of a vaccine. The critical determinant of the protection duration time is the antibody decay rate, and this rate of decay is closely related to the antigenlike substances in the environment. More importantly, this antibody decay rate is eventually dependent on the properties of the antibody itself. We should target the induction of antibodies with FAST binding activity. It is important to note that the decay rate is not equivalent to half-life because almost all immune cells have the same half-life regardless of what antibody it expresses. The main factor contributing to the difference in decay rate is that different antibodies will be affected differently by environmental antigen-like substances.

Finally, our model also shows that the peak of the immune response is exceptionally weakly affected by the number of initial invading viruses, but the number of initial invading viruses significantly affects the latency time of the virus. Our antibody dynamic model strongly supports our UTR deletion theory in COVID-19 infection [37]. Nevertheless, we also have to admit there are lots of hypotheses and uncertainties in our model. Furthermore, it still lacks an in-vivo experiment data fitting process, while many of the predictions and theories in the article have remained to be confirmed experimentally and statistically in the future.

\section{Supplementary materials}

All supplementary materials and Matlab codes can be accessed at: https://github.com/zhaobinxu23/antibody_dynamics 


\section{Declaration of Interest}

We declare that this manuscript is original, has not been published before and is not currently being considered for publication elsewhere.

We know of no conflicts of interest associated with this publication, and there has been no significant financial support for this work that could have influenced its outcome. As Corresponding Author, I confirm that the manuscript has been read and approved for submission by all the named authors.

\section{Reference.}

[1] Skegg D, Gluckman P, Boulton G, et al. Future scenarios for the COVID-19 pandemic[J]. The Lancet, 2021, 397(10276): 777-778.

[2] Xu Z, Zhang H. If we cannot eliminate them, should we tame them? Mathematics underpinning the dose effect of virus infection and its application on covid-19 virulence evolution[J]. medRxiv, 2021.

[3] Xu Z, Zhang H, Niu Y. A Continuous Bayesian Model for the Stimulation COVID-19 Epidemic Dynamics[J]. medRxiv, 2021.

[4] Sadoff J, Gray G, Vandebosch A, et al. Safety and efficacy of single-dose Ad26. COV2. S vaccine against Covid-19[J]. New England Journal of Medicine, 2021, 384(23): 2187-2201.

[5] Klugar M, Riad A, Mekhemar M, et al. Side effects of mRNA-based and viral vector-based COVID-19 vaccines among German healthcare workers[J]. Biology, 2021, 10(8): 752.

[6] Riad A, Pokorná A, Attia S, et al. Prevalence of COVID-19 Vaccine Side Effects among Healthcare Workers in the Czech Republic[J]. Journal of Clinical Medicine, 2021, 10(7): 1428.

[7] Klein S L, Shann F, Moss W J, et al. RTS, S malaria vaccine and increased mortality in girls[J]. 2016.

[8] Aaby P, Mogensen S W, Rodrigues A, et al. Evidence of increase in mortality after the introduction of diphtheria-tetanus- pertussis vaccine to children aged 6-35 months in Guinea-Bissau: a time for reflection?[J]. Frontiers in public health, 2018, 6: 79.

[9] Hunter P R, Brainard J S. Estimating the effectiveness of the Pfizer COVID-19 BNT162b2 vaccine after a single dose. A reanalysis of a study of 'real- world' vaccination outcomes from Israel[J]. Medrxiv, 2021.

[10] Smith A M, Adler F R, McAuley J L, et al. Effect of 1918 PB1-F2 expression on influenza A virus infection kinetics[J]. PLoS computational biology, 2011, 7(2): e1001081.

[11] Baccam P, Beauchemin C, Macken C A, et al. Kinetics of influenza A virus infection in humans[J]. Journal of virology, 2006, 80(15): 7590-7599.

[12] Beauchemin C A A, McSharry J J, Drusano G L, et al. Modeling amantadine treatment of influenza A virus in vitro[J]. Journal of theoretical biology, 2008, 254(2): 439-451.

[13] Handel A, Longini Jr I M, Antia R. Neuraminidase inhibitor resistance in influenza: assessing the danger of its generation and spread [J]. PLoS Computational Biology, 2007, 3(12): e240.

[14] Canini L, Perelson A S. Viral kinetic modeling: state of the art[J]. Journal of pharmacokinetics and pharmacodynamics, 2014, 41(5): 431-443.

[15] Hancioglu B, Swigon D, Clermont G. A dynamical model of human immune response to influenza A virus infection[J]. Journal of theoretical biology, 2007, 246(1): 70-86.

[16] Handel A, Longini Jr I M, Antia R. Towards a quantitative understanding of the within-host dynamics of influenza A infections[J]. Journal of the Royal Society Interface, 2010, 7(42): 35-47.

[17] Lee H Y, Topham D J, Park S Y, et al. Simulation and prediction of the adaptive immune response to influenza A virus infection[J]. Journal of virology, 2009, 83(14): 7151-7165.

[18] Miao H, Hollenbaugh J A, Zand M S, et al. Quantifying the early immune response and adaptive immune response kinetics in mice infected with influenza A virus[J]. Journal of virology, 2010, 84(13): 6687-6698.

[19] Tridane A, Kuang Y. Modeling the interaction of cytotoxic T lymphocytes and influenza virus infected epithelial cells[J]. Mathematical Biosciences \& Engineering, 2010, 7(1): 171.

[20] Clapham H E, Quyen T H, Kien D T H, et al. Modelling virus and antibody dynamics during dengue virus infection suggests a role for antibody in virus clearance[J]. PLoS computational biology, 2016, 12(5): e1004951.

[21] Best K, Guedj J, Madelain V, et al. Zika plasma viral dynamics in nonhuman primates provides insights into early infection and antiviral strategies [ J]. Proceedings of the National Academy of Sciences, 2017, 
114(33): 8847-8852.

[22] Owen J A, Punt J, Stranford S A. Kuby immunology [M]. New York, NY, USA:: WH Freeman, 2013. [23] Marín-Hernández D, Schwartz R E, Nixon D F. Epidemiological evidence for association between higher influenza vaccine uptake in the elderly and lower COVID-19 deaths in Italy[J]. Journal of medical virology, 2020.

[24] Salem M L, El-Hennawy D. The possible beneficial adjuvant effect of influenza vaccine to minimize the severity of COVID-19 [J]. Medical hypotheses, 2020, 140: 109752.

[25] Fink G, Orlova-Fink N, Schindler T, et al. Inactivated trivalent influenza vaccination is associated with lower mortality among patients with COVID-19 in Brazil[J]. BMJ evidence-based medicine, 2021, 26(4): 192-193.

[26] Escobar L E, Molina-Cruz A, Barillas-Mury C. BCG vaccine protection from severe coronavirus disease 2019 (COVID-19) [J]. Proceedings of the National Academy of Sciences, 2020, 117(30): 17720-17726.

[27] Huang D, Tran J T, Olson A, et al. Vaccine elicitation of HIV broadly neutralizing antibodies from engineered B cells[J]. Nature communications, 2020, 11(1): 1-10.

[28] Voss J E, Gonzalez-Martin A, Andrabi R, et al. Reprogramming the antigen specificity of B cells using genome-editing technologies[J]. Elife, 2019, 8: e42995.

[29] Moffett H F, Harms C K, Fitzpatrick K S, et al. B cells engineered to express pathogen-specific antibodies protect against infection [J]. Science immunology, 2019, 4(35).

[30] Azam M, Sulistiana R, Ratnawati M, et al. Recurrent SARS-CoV-2 RNA positivity after COVID-19: a systematic review and meta-analysis[J]. Scientific reports, 2020, 10(1): 1-12.

[31] Dao T L, Hoang V T, Gautret P. Recurrence of SARS-CoV-2 viral RNA in recovered COVID-19 patients: a narrative review[J]. European Journal of Clinical Microbiology \& Infectious Diseases, 2021, 40(1): $13-25$.

[32] Kang M, Xin H, Yuan J, et al. Transmission dynamics and epidemiological characteristics of Delta variant infections in China[J]. medRxiv, 2021.

[33] Heneghan C, Brassey J, Jefferson T. SARS-CoV-2 viral load and the severity of COVID-19 [J]. 2020.

[34] Gandhi M, Beyrer C, Goosby E. Masks do more than protect others during COVID-19: reducing the inoculum of SARS-CoV-2 to protect the wearer[J]. Journal of general internal medicine, 2020, 35(10): 30633066.

[35] Spinelli M A, Glidden D V, Gennatas E D, et al. Importance of non-pharmaceutical interventions in lowering the viral inoculum to reduce susceptibility to infection by SARS-CoV-2 and potentially disease severity[J]. The Lancet Infectious Diseases, 2021.

[36] Bielecki M, Züst R, Siegrist D, et al. Social distancing alters the clinical course of COVID-19 in young adults: a comparative cohort study[J]. Clinical infectious diseases, 2021, 72(4): 598-603.

[37] 